\title{
Coding of Serial Order in Verbal, Visual and Spatial Working Memory
}

\author{
Véronique Ginsburg and Kim Archambeau \\ Université Libre de Bruxelles
}

\author{
Jean-Philippe van Dijck \\ Ghent University and Thomas More University College
}

\author{
Fabienne Chetail and Wim Gevers \\ Université Libre de Bruxelles
}

\begin{abstract}
In the domain of working memory, recent theories postulate that the maintenance of serial order is driven by position marking. According to this idea, serial order is maintained though associations of each item with an independent representation of the position that the item constitutes in the sequence. Recent studies suggest that those position markers are spatial in nature, with the beginning items associated with left side and the end elements with the right side of space (i.e., the ordinal position effect). So far however, it is unclear whether serial order is coded along the same principles in the verbal and the visuospatial domain. The aim of the current study was to investigate whether serial order is coded in a domain general fashion or not. To unravel this question, 6 experiments were conducted. The first 3 experiments revealed that the ordinal position effect is found with verbal but not with spatial information. In the subsequent experiments, the authors isolated the origin of this dissociation and conclude that to obtain spatial coding of serial order, it is not the nature of the encoded information (verbal, visual, or spatial) that is crucial, but whether the memoranda are semantically processed or not. This work supports the idea that serial order is coded in a domain general fashion, but suggests that position markers are only spatially coded when the to-be-remembered information is processed at the semantic level.
\end{abstract}

Keywords: serial order, working memory, space, semantic, position marking

Working memory (WM) is a fundamental cognitive function that refers to the brief maintenance of information in an active and accessible state such that operations can be performed on it. It is considered to be crucial for several major cognitive skills such as reasoning, calculation, language, or learning (Baddeley \& Hitch, 1998; see also Repovs \& Baddeley, 2006). Besides the storage of information, an important feature of WM is its ability to encode and maintain information about the serial order of perceived events. Many researchers have studied the mechanisms by which serial order is coded and retrieved (Ebbinghaus, 1885/1964; Lash-

Véronique Ginsburg and Kim Archambeau, Center for Research in Cognition and Neurosciences, Université Libre de Bruxelles; Jean-Philippe van Dijck, Department of Experimental Psychology, Ghent University, and Department of Clinical Psychology and Department of Applied Psychology, Thomas More University College; Fabienne Chetail and Wim Gevers, Center for Research in Cognition and Neurosciences, Université Libre de Bruxelles.

Véronique Ginsburg and Kim Archambeau contributed equally to the article, therefore are combined first coauthors. Véronique Ginsburg and Kim Archambeau were Research Fellows at the Belgian Fonds National de la recherche Scientifique (FNRS) when the study was conducted. A subset of the results discussed in this article were presented at two conferences (BAPS, 2014, Brussels, Belgium and the European Workshop on Cognitive Neuropsychology, 2015, Bressanone, Italy). No other formats were used to share this work.

Correspondence concerning this article should be addressed to Véronique Ginsburg, Center for Research in Cognition and Neurosciences, Université Libre de Bruxelles, Avenue Franklin Roosevelt 50, B-1050 Brussels, Belgium. E-mail: veronique.ginsburg@ulb.ac.be ley, 1951; Sternberg, 1967; for a review see Marshuetz, 2005) and several hypotheses have emerged. Associative chaining theory was one of the earliest approaches trying to explain how serial order is coded in WM (e.g., Ebbinghaus, 1885/1964). The basic idea underlying chaining theory is that serial order is maintained by creating associations between items. This implies that each recalled item becomes the cue for the next item. Chaining models are very efficient in explaining a hallmark observation in serial order: the gradual increase in reaction time (RT) when retrieving order information further away in the memorized sequence (e.g., Sternberg, 1975). However, several immediate objections to chaining models emerged. Chaining models have difficulties to explain typically observed error patterns. For instance, chaining models assume that serial order is encoded by contiguous associations between items. Therefore, recall should fail for all items following an erroneous recall. This is not what is typically observed (e.g., Henson, Norris, Page, \& Baddeley, 1996). Furthermore, chaining models also predict that response latencies should be faster for items closer in the memorized sequence. However, this is the opposite of what is observed (e.g., Marshuetz, Smith, Jonides, DeGutis, \& Chenevert, 2000). In this study, participants had to judge whether two items were in the same order as in a memorized sequence. Response latencies increased the smaller the distance between the two memorized items. For these (and other) reasons, theorists gradually rejected the hypothesis that chaining plays a dominant role in serial order memory (e.g., Burgess \& Hitch, 2006; Farrell \& Lewandowsky, 2002; Henson et al., 1996).

Other, so-called positional models suggest that item and order information in memory are represented and processed separately (Henderson \& Matthews, 1970; McElree, Dosher, 1993; Sternberg, 1967). It is 
argued that serial order memory is driven by item marking (i.e., associations or bindings between items) and some independent representation of order (i.e., time, temporal context, or ordinal list position; Lewandowsky \& Farrell, 2008). The idea of position coding was put forward on the basis of theoretical ground but strong empirical support was until recently lacking. Recent fMRI evidence more directly favors the idea of position marking (Kalm \& Norris, 2014), although the exact nature of the markers remains debated.

Besides the question on how serial order is coded, another outstanding question is whether the mechanisms for coding serial order in WM are domain general (e.g., across the verbal and the visuospatial domains) or not. Although consensus is not yet reached, some indications support the hypothesis of a domaingeneral mechanism (for a review see Hurlstone, Hitch, \& Baddeley, 2014). Some observed that several behavioral signatures of serial order coding are similar in verbal and spatial short-term memory (STM). For example, Jones and colleagues (1995) demonstrated that the pattern of errors was equivalent for spatial and verbal tasks (e.g., increase of errors with list length, primacy and recency effects). Others observed that verbal and spatial serial order (but not item) in WM interferes with each other if one type of information has to be processed within the retention interval of the other (Depoorter \& Vandierendonck, 2009). Note that these observations are indirect, because a similar pattern of data can result from different underlying cognitive mechanisms, and the interference can be caused by shared peripheral processes that are not intrinsically linked to serial order coding (e.g., the processing of sequential vs. simultaneous information; Cornoldi \& Vecchi, 2004). In addition, it has been observed that visuospatial stimuli can be stored using a verbalization strategy (using the phonological loop) or not (Rothmayr, Baumann, Endestad, Rutschmann, Magnussen, \& Greenlee, 2007). This opens the additional possibility that the observed behavioral similarities between verbal and spatial serial order in WM are caused by the use of a common verbal strategy rather than by shared coding mechanisms. In sum, to provide a final answer to the question about domain-generality, direct behavioral evidence is needed.

Recently a new suggestion to account for serial order coding in WM was proposed (Oberauer, 2009). In this theory it is suggested that items that have to be maintained and processed in WM are represented as increased activations in long-term memory. This increased activation would make these items directly accessible through temporary bindings established between items (e.g., objects, words) and contexts (i.e., positions in a generic cognitive coordinate system). These temporary bindings link the items with locations in mental space such as the ordinal position in a list. This idea gained empirical support by the observation that serial order coding of verbal information in memory is spatially organized. That is, a systematic association between the ordinal position of an item in verbal WM and response side was observed when retrieving memorized information. These observations were obtained thanks to a new research design mixing a memory task with a classification task (e.g., Ginsburg, van Dijck, Previtali, Fias, \& Gevers, 2014; Guida, Leroux, Lavielle-Guida, \& Noël, 2016; van Dijck \& Fias, 2011). In this paradigm, participants have to memorize a sequence of five random digits visually presented while performing a parity judgment task. Importantly, participants have to classify the digit as odd or even only if the item belongs to the memorized sequence (go-nogo procedure). This go-nogo procedure ensures that memorized numbers have to be retrieved from WM. During the parity judgment task, it was observed that numbers from the beginning of the memorized sequence were responded to faster with the left-hand side whereas numbers at the end of the sequence were responded to faster with the right-hand side (van Dijck \& Fias, 2011). From here on this association between a spatial response and the ordinal position in WM is termed the ordinal position effect (e.g., Ginsburg et al., 2014). Note that the same interaction between space and order information processing has also been termed SPoARC (Spatial-Positional Association of Response Codes), referring to exactly the same phenomenon (Guida \& Guida-Lavielle, 2014; see also Guida et al., 2016). Van Dijck and Fias (2011) ran a subsequent experiment to demonstrate that these spatial-position associations are not limited to numerical information but can be extended to other verbal material as well. This time, participants classified words that were memorized in WM as fruits or vegetables. Again, the ordinal position effect was observed. Fruits or vegetables early in the sequence were responded to faster with the left-hand side and fruits or vegetables late in the sequence were responded to faster with the right-hand side. Recently, this ordinal position effect has also been replicated with auditory presentation of items (i.e., consonants) in WM (Guida et al., 2016). In this study, participants had to perform a slightly different task by pressing a "yes" or a "no" key to indicate whether the heard consonant was part of the memorized sequence or not. Yes responses with the left hand were initiated faster for items in the beginning of the sequence, whereas yes responses with the right hand were faster for items at the end of the sequence. In other words, the ordinal position effect is observed not only using go-nogo task paradigms but is also observed in more straightforward recognition tasks. The strength of such designs (Ginsburg et al., 2014; Guida et., 2016; van Dijck \& Fias, 2011) is that they provide with a direct behavioral signature to investigate the nature of serial order coding in WM. Bringing together positional models and observations of the ordinal position effect, Abrahamse, van Dijck, Majerus, and Fias (2014) proposed the mental whiteboard hypothesis to explain serial order coding in WM. This hypothesis is in line with serial order models suggesting that each element in WM is associated with an independent representation of its ordinal position in the memorized sequence through positional markers. Moreover, the mental whiteboard hypothesis specifies that those positional markers are spatial in nature.

The question addressed here is whether serial order in WM is spatially coded for all types of information. In a first step (Experiments 1,2 , and 3 ), we investigate whether the ordinal position effect reflecting spatial coding of serial order in WM is domainspecific (established only with verbal material) or domain-general by using the paradigm of van Dijck and Fias (2011) with spatial material. To anticipate on the results, in those first three experiments a clear dissociation was observed between verbal and spatial serial order processing in WM: The ordinal position effect was observed with verbal but not with spatial information. At this point, we suggested that serial order is maintained spatially in WM only for verbal materials (domain-specific). Experiment 4 was designed to investigate the importance of verbalization for the ordinal position effect. Finally, Experiments 5 and 6 investigated 
the relative importance of verbalization but also of semantic activation to observe spatial coding of serial order in WM.

\section{Experiment 1}

Recently, van Dijck and Fias (2011) reported an association between the ordinal position of verbal elements (i.e., words) in WM (i.e., ordinal position effect) and lateralized responses. Here, we first wanted to replicate this observation with word material to ensure the reproducibility of this effect with verbal information (verbal condition). Second, we investigated whether the spatial coding of verbal serial memorized information could be extended to the spatial domain. To do so, the task of van Dijck and Fias (2011) was adapted such that participants memorized and classified spatial information.

\section{Participants}

Thirty-five undergraduate students ${ }^{1}$ from the Université Libre de Bruxelles (ULB) received course credits to participate in the study. They were all at least 18 years old (on average 21.11 years, $S D=2.99$ ). There were 28 females (one left-handed) and 7 males (one left-handed). In this experiment (as well in the following ones), the local ethical committee approved this study and participants received a debriefing after completing two sessions of 30 min, on two consecutive days. All participants were naive with respect to the purpose of the experiment.

\section{Materials}

Two different sets of stimuli were constructed for the verbal and the spatial conditions. The verbal set consisted of 8 high frequency French words. As in the experiment of van Dijck and Fias (2011), there were 4 fruits (pomme, apple; fraise, strawberry; melon, melon; and prune, plum) and 4 vegetables (oignon, onion; laitue, lettuce; poireau, leek; navet, turnip]. The spatial set consisted of a random selection of 8 black dots (filled circles) from a not visible matrix composed by an $8 \times 8$ array of squares.

\section{Stimuli and Procedure}

Every participant performed both conditions (in different sessions) and the order of conditions was counterbalanced across participants.

\section{Verbal Condition}

The experiment was performed using E-Prime 2 Professional Software (Psychology Software Tools). Participants were seated in a quiet room approximately $50 \mathrm{~cm}$ from a 17 -inch LCD computer screen with a resolution of $1280 \times 1024$ pixels. Motor responses were collected via button presses on a response box. Each word (approximately 35 by $5 \mathrm{~mm}$ on the screen) was presented on the computer screen in white color on a black background.

The condition consisted of 16 blocks. Each block was divided in three subsequent phases: an encoding phase, a classification phase and a control phase. During the encoding phase, 4 words ( 2 fruits and 2 vegetables) were successively presented at the center of the screen, in a random order. New sequences were generated every block, but care was taken that over the entire experiment, each word was presented on each WM position an equal amount of times. Participants were instructed to memorize this sequence of words in the serial order of presentation. To enable successful encoding, participants pressed a button on the response box to proceed from one word to the next.

A blank screen followed the final word $(2500 \mathrm{~ms})$ allowing for rehearsal, after which the classification phase started. This period of rehearsal was important to maximize the chance that all four elements were correctly maintained in the order of presentation during the entire block. During this classification phase, while keeping the memorized sequence in mind, all eight words from the set (including the four memorized words) were randomly presented twice with the restriction that the same word could not be repeated on consecutive trials. The task was to determine if the presented word was a fruit or a vegetable but participants had to respond only to words that were part of the memorized sequence (go-nogo paradigm).

A trial consisted of a fixation point $(500 \mathrm{~ms})$ followed by a presented word. If this word belonged to the memorized sequence, participants had to classify it as fruit or vegetable, as quickly as possible, by pressing a left or a right-sided button. The response deadline was set to $1500 \mathrm{~ms}$. After this deadline or after a response, the next trial was initiated, following an intertrial interval of $1000 \mathrm{~ms}$. One half of the participants had to press the left button for 'fruits' and the right button for 'vegetables', whereas for the other half this response mapping was reversed.

During the last phase (the control phase to make sure that participants had correctly maintained the memorized sequence in the correct order), three couples of words were presented successively on the screen. For each couple of words, participants had to indicate if the first word of the pair was presented before or after the second word of the pair in the memorized sequence. Participants had to press on the letter "o" if the first word was presented before the second and on the letter "n" if it was not the case. The words presented in each pair were always neighboring items in the WM sequence.

A given block was introduced again at the end of the experiment if the participant responded erroneously to at least one of the three pairs.

\section{Spatial Condition}

The experiment was performed using the Psychtoolbox extension (Brainard, 1997) running with Matlab (ver. R2010a, The Mathworks Inc., Natick, Massachusetts, U.S.A.). The computer screen had a resolution of $1280 \times 1024$ pixels. The motor responses were collected via a keyboard. The size of each dot was approximately 27 pixels.

\footnotetext{
${ }^{1}$ A priori power analyses were conducted on the basis of Ginsburg et al. (2014). The sample required to have a power of .80 was 15 and 16 if we take into account the ordinal position effect based on the regression analysis of the first and the second experiment of that study respectively. On the basis of this, it was decided to test minimally 25 participants in total sample of each experiment. Moreover, the groups in experiments with between-factor designs were always bigger than 15-16 participants required by the a priori power analyses.
} 
The experimental design was similar to the one of the verbal condition with the crucial difference that words were replaced by spatial dots. During the encoding phase, 4 black dots (positioned randomly in a nonvisible matrix) were successively presented (600 $\mathrm{ms}$ per dot). Participants were instructed to memorize this sequence of dots in the correct order. To enable efficient encoding, participant could press, four times maximum, on a button to visualize the sequence again.

During the classification phase, eight dots (containing the four memorized dots) were randomly presented twice, once with a white line which passes through the circle inclined at $30^{\circ}$ and once with a white line inclined at $75^{\circ}$. Participants had to classify (by pressing a left or a right button) the inclination of the white line only if the presented dot belonged to the memorized sequence. As in the verbal condition, the task consisted of 16 blocks with each response mapping counterbalanced across participants.

During the last phase, the control phase, to make sure that participants had correctly maintained the memorized sequence in the correct order, the eight dots were presented simultaneously on the screen and participants had to click with a mouse to the four memorized dots in the correct order. The block was introduced again at the end of the experiment if the participant responded erroneously.

\section{Data Analysis}

Across conditions (verbal, spatial), we used repeated measures ANOVA with ordinal position in WM (4: 1 to 4 ) and response side (2: left, right) as within-subjects factors. On the basis of these factors, we could investigate the interaction between ordinal position and response side (ordinal position effect) and the main effect of ordinal position (serial search effect). This analysis was complemented by using the regression linear approach described by Lorch and Myers (1990, see also Fias, Brysbaert, \& Geypens, 1996). This method consists of computing the difference in RTs (dRT; RT right hand minus RT left hand) for each position in WM (from 1 to 4 ) separately. For each participant, these values were entered in a regression analysis with position as predictor. A $t$ test was performed to evaluate whether the regression weights of the group deviated significantly from zero. Because of the characteristics of the ordinal position effect (faster left-hand responses for early items in the memorized sequence and faster right-hand responses for late items in the memorized sequence), a negative trend in the RT difference ( $\mathrm{dRT}$ ) values is expected the further the position in the sequence. Throughout the manuscript, all reported $p$ values are 2-sided. $p$ values falling between .05 and .1 are described as 'just failing to reach significance.' Whenever such results are obtained, we refrain from making strong conclusions or further exploration is performed in subsequent experiments. Finally, a Bayesian analysis (Eidswick, 2012; see also Wagenmakers, Wetzels, Borsboom, \& van der Maas, 2011 for an interpretation of Bayes-factors) was conducted on regression slopes in order to corroborate the presence of ordinal position effects and/or to confirm their absence (null hypothesis).

\section{Results and Discussion}

Across the two conditions (verbal and spatial), three participants were excluded from the analysis. Two of them made too many errors (more than 2 SDs above the mean of errors) -one in the verbal condition and the other in the spatial condition-, and another responded too slowly (more than $2 S D$ s above the mean of the RTs) in the verbal condition. In both conditions, we only took into account blocks with a correct control phase (16 blocks) and go-nogo trials with correct RTs (RTs) larger than $250 \mathrm{~ms}$.

Verbal condition. It took on average 19.59 blocks $(S D=$ 3.26) before all 16 blocks were correctly performed. During the classification phase, average RT was $713.50 \mathrm{~ms}(S D=78.68 \mathrm{~ms})$ and the average number of errors was $7.64 \%(S D=3.86 \%)$.

The WM Position $\times$ Response Side $(4 \times 2)$ repeated measures ANOVA revealed a main effect of WM position $[F(3$, $\left.93)=12.54, p<.001, \eta_{P}^{2}=.29\right]$. Average RTs per position increased gradually $(686,711,724,734 \mathrm{~ms}$, for each position respectively). A polynomial contrast confirmed the linear trend of these RTs $\left[F(1,31)=46.53, p<.001, \eta_{P}^{2}=.60\right]$ indicating that the serial order of the sequence was processed. Importantly, the interaction between $\mathrm{WM}$ position and response side $[F(3$, $\left.93)=3.33, p<.05, \eta_{P}^{2}=.10\right]$ was also significant. We further evaluated this interaction by using the linear regression approach described by Lorch and Myers (1990; see also Fias et al., 1996). This regression analysis confirmed the presence of an ordinal position effect with the difference between right- and left-hand responses decreasing with $16.22 \mathrm{~ms}$ per position $[\mathrm{dRT}=-16.22 \mathrm{~ms}, t(31)=-3.02, p<.01]$ (Figure 1A). Finally, a Bayesian independent samples $t$ test on the regression slopes resulted in a Bayes-factor (BF) of 7.96 providing substantial evidence for the presence of an ordinal position effect with verbal information.

Spatial condition. It took on average 19.69 blocks ( $S D=$ 3.02) before all 16 blocks were correctly performed. During the classification phase, average RT was $737.22 \mathrm{~ms}(S D=76.12 \mathrm{~ms})$ and the average number of errors was $7.67 \%(S D=4.28 \%)$.

The WM Position $\times$ Response Side $(4 \times 2)$ repeated measures ANOVA revealed a significant main effect of WM position $[F(3$, $\left.93)=11.57, p<.001, \eta_{P}^{2}=.27\right]$. Average RTs per position increased gradually $(724,731,735,760)$. A polynomial contrast confirmed the linear trend of these RTs $[F(1,31)=28.38, p<$ $\left..001, \eta_{P}^{2}=.48\right]$. In contrast to the verbal condition, the interaction between ordinal position and response side was not significant, indicating the absence of the ordinal position effect $[F(3,93)=$ $\left..28, p=.84, \eta_{P}^{2}<.01\right]$. The linear regression approach analysis (Lorch \& Myers, 1990; Fias et al., 1996) also failed to show the presence of the ordinal position effect [slope $=3.56(S D=32.32)$; $t(31)=.61, p=.54]$ (Figure 1B). In order to confirm that this null result denotes an absence of ordinal position effect (null hypothesis), we performed a Bayesian independent samples $t$ test on the regression slopes. A BF of 0.23 was observed providing substantial evidence for the null hypothesis, confirming the absence of an ordinal position effect with spatial information.

We replicated previous work (van Dijck \& Fias, 2011), both the ordinal position effect and the serial search effect being observed with verbal material. Surprisingly, this association was not observed when participants classified spatial information, suggesting the existence of a dissociation. This dissociation is further confirmed when directly comparing the linear trends (regression slopes) reflecting the ordinal position effect in the verbal and in the spatial condition: The linear trends significantly differed from each other with a more negative 

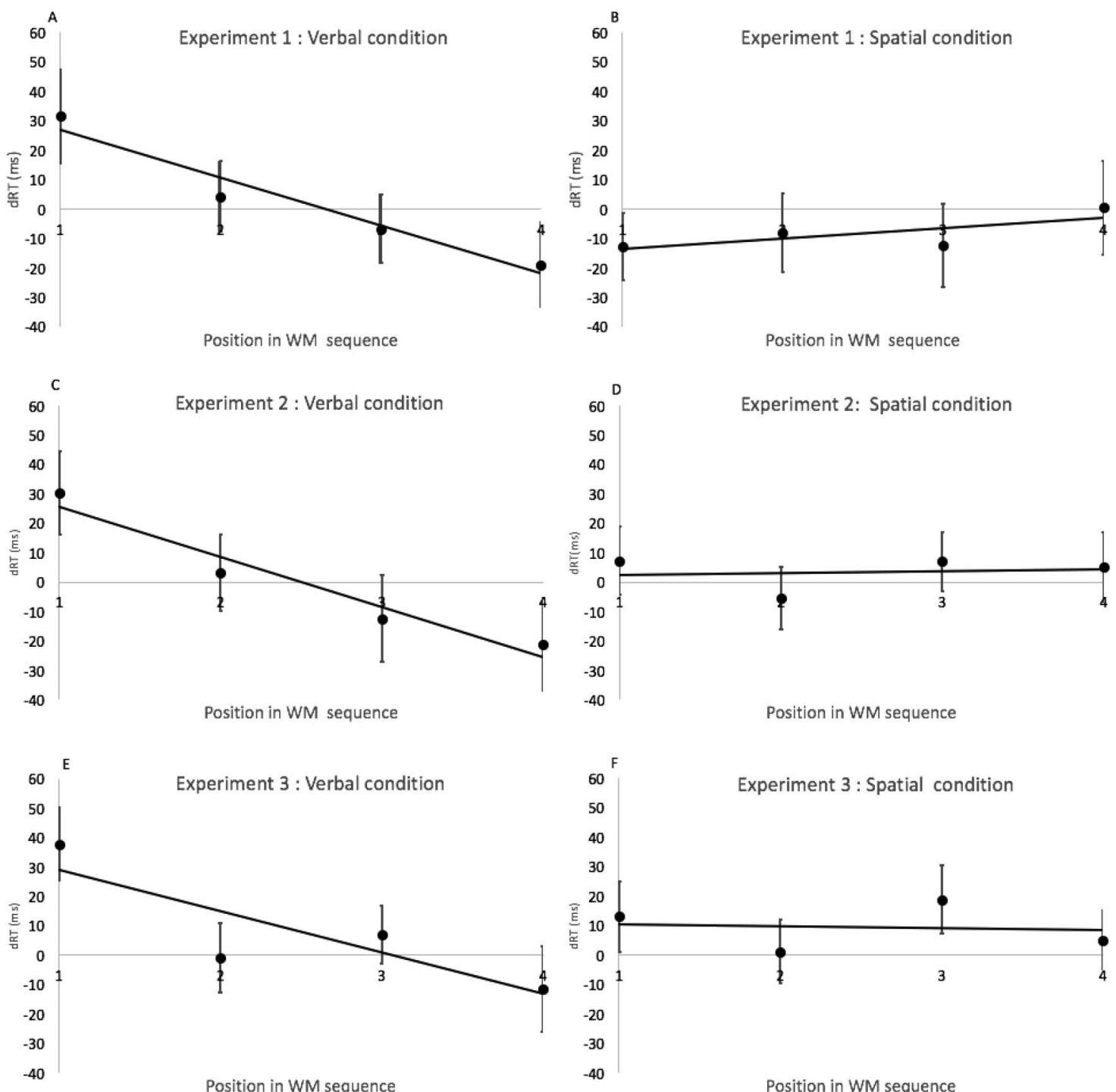

Figure 1. The ordinal position effect with verbal and spatial materials. Panel A and B depict the results of the verbal and spatial condition of Experiment 1. Panel $\mathrm{C}$ and $\mathrm{D}$ depict the results of the verbal and spatial conditions of Experiment 2. Panel E and F depict the results of the verbal and spatial conditions of Experiment 3. The Y-axis represents difference in reaction times (dRTs). These are obtained by subtracting the average RTs from the right hand with the average RTs from the left hand. Each point reflects the dRT for a position in the working memory (WM) sequence. If a dRT has a positive value, left-hand responses are faster than right-hand responses. The black line is obtained after regressing the $\mathrm{dRT}$ to the positions in the sequence. A line with a negative slope is indicative for the expected relation between serial order in WM and space.

slope for the verbal compared to the spatial condition $[-16.22$ versus $3.56 ; t(31)=-2.41, p<.05]$. A Bayesian paired samples $t$ test with the spatial and verbal regression slopes was conducted. A BF of 2.27 was obtained suggesting anecdotal evidence for a difference in ordinal position effect between the spatial and the verbal condition.

\section{Experiment 2}

The purpose of Experiment 2 is twofold. The first aim is the replication of the results of Experiment 1, demonstrating an ordinal position effect when verbal information but not when spatial information has to be classified in WM. As it is known that encoding time plays an important role in WM functioning (e.g., Barrouillet, Plancher, Guida, \& Camos, 2013), the second aim is to test whether timing differences in the encoding of verbal and spatial information in Experiment 1 caused the absence of the ordinal position effect in the spatial condition. In Experiment 1, all dots were presented rapidly in a sequence after which the entire sequence could be repeated while participants proceeded from one verbal item to the next at their own pace. Therefore, in Experiment 2, participants again performed both a 
verbal and a spatial condition, but in both conditions the stimuli were encoded in exactly the same way with participants proceeding from one item to the next at their own pace.

\section{Participants}

Thirty-four new undergraduate students from the Université Libre de Bruxelles received course credits to participate in the study. All participants were at least 18 years old (on average 19.62 years, $S D=1.74$ ). There were 28 females (two left-handed) and 6 males (one left-handed).

\section{Material, Stimuli, and Procedure}

We used the same material, stimuli, and procedure as in Experiment 1 , for both conditions (verbal and spatial). We only replaced, in the spatial condition, the encoding deadline of $600 \mathrm{~ms}$ per dots by a self-paced presentation. Consequently, as in the verbal condition, participants had to press the space bar to proceed from one dot to another during the encoding phase. Every participant performed both conditions (in different sessions) and the order of conditions was counterbalanced across participants.

\section{Results and Discussion}

Across the two conditions (verbal and spatial), four participants were excluded from the analysis. Two of them-one in the verbal condition and the other in the spatial condition-made too many errors (more than $2 S D$ s above the mean of errors) and two others-one in the verbal condition and the other in the spatial condition-responded too slowly (more than $2 S D$ s above the mean of the RTs).

Verbal condition. In this condition, it took on average 19.94 blocks ( $S D=4.66$ ) before all 16 blocks were correctly performed. During the classification phase, average RT was $727.20 \mathrm{~ms}(S D=$ $100.40 \mathrm{~ms})$ and the average number of errors was $9.12 \%(S D=$ $4.30 \%)$.

The WM Position $\times$ Response Side $(4 \times 2)$ repeated measures analysis of variance (ANOVA) indicated a main effect of WM position $\left[F(3,87)=4.22, p<.01, \eta_{P}^{2}=.13\right]$. Average RTs per position indicated a gradual increase $(700,707,724,734 \mathrm{~ms}$ for each position, respectively). A polynomial contrast confirmed the linear trend of these RTs, $F(1,29)=9.52, p<.005, \eta_{P}^{2}=.25$, suggesting that the serial order of the items was processed. Replicating the ordinal position effect, a significant interaction was observed between WM position and response side, $F(3,87)=$ 2.84, $p<.05, \eta_{P}^{2}=.01$. The regression analysis confirmed an association of the initial elements with the left-hand side and the final elements with the right-hand side, as dRTs decreased per position with $16.99 \mathrm{~ms}$ per position, $t(29)=-3.04, p<.01$ (Figure 1C). The Bayesian independent samples $t$ test on the regression slopes resulted in a $\mathrm{BF}$ of 8.21 providing substantial evidence for the presence of an ordinal position effect with verbal information.

Spatial condition. It took on average 20.10 blocks $(S D=$ 3.61) before all 16 blocks were correctly performed. During the classification phase, average RT was $764.54 \mathrm{~ms}(S D=88.37 \mathrm{~ms})$ and the average number of errors was $7.85 \%(S D=5.86 \%)$.

The WM Position $\times$ Response Side $(4 \times 2)$ repeated measures ANOVA indicated a significant main effect of WM position
$\left[F(3,87)=11.53, p<.001, \eta_{P}^{2}=.28\right]$. Average RTs per position increased gradually $(731,733,742,769 \mathrm{~ms}$ for each position, respectively). A polynomial contrast confirmed the linear trend of these RTs $\left[F(1,29)=39.68, p<.001, \eta_{P}^{2}=.58\right]$. As in the spatial condition of Experiment 1, the interaction between WM position and response was not significant $\left[F(3,87)=.42, p=.74, \eta_{P}^{2}=\right.$ .014]. Finally, the regression analysis also failed to confirm the presence of the ordinal position effect [slope $=.62 ; t(29)=0.12$, $p=.90]$ (Figure 1D). A BF of 0.20 was observed with the Bayesian independent samples $t$ test on spatial regression slopes. In other words, there is substantial evidence for the absence of an ordinal position effect with spatial information.

Taken together, the results of Experiment 1 were replicated when encoding conditions were exactly matched for the spatial and the verbal task. In addition, as in Experiment 1, the linear trends for the verbal and the spatial condition dissociated, with a more negative slope for the verbal compared to the spatial condition, -16.99 versus $0.62 ; t(29)=-2.72, p<.05$. In agreement with the inferential analyses, a Bayesian paired samples $t$ test resulted in a $\mathrm{BF}$ of 4.21 providing substantial evidence for a difference in ordinal position effect between the spatial and the verbal condition.

\section{Experiment 3}

The first aim of Experiment 3 was to confirm the findings of Experiments 1 and 2 (i.e., an ordinal position effect with verbal but not with spatial information). This is important because we wanted to ensure that the observed dissociation between spatial and verbal processing was not the result of systematic methodological differences between both conditions. Indeed, the categorization and control phases used in previous experiments differed between the verbal and the spatial condition. For instance, although participants classified words according to category (e.g., fruits or vegetables) they classified positions with orientation discriminations (e.g., a line tilted to the left or to the right). In Experiment 3, participants again performed both a verbal and a spatial condition but this time, the experimental design for both conditions was identical and differed only in the nature of the material encoded and maintained in WM.

\section{Participants}

Thirty-four new undergraduate students from the Université Libre de Bruxelles received course credits to participate in the study. They were all at least 18 years old (on average 22.26 years, $S D=3.44$ ). There were 26 females (four left-handed) and eight males (two left-handed).

\section{Material}

In the spatial condition we used the same material as in the previous experiments. In the verbal condition, we replaced the eight words (four fruits and four vegetables) by a set of 64 words corresponding to concrete drawings (which will again be used in Experiment 4). This enabled us to design a color go-nogo task.

\section{Stimuli and Procedure}

The procedure for the verbal and spatial condition was identical and differed only in the type of stimuli used (i.e., words vs. spatial 
positions). For both conditions, $16 \mathrm{WM}$ sequences were presented in total. During the encoding phase, participants had to memorize four items (presented in black-and-white) presented one after the other for 3,500 ms. During the categorization phase, eight itemsincluding the four memorized items-were presented. Each item appeared randomly twice, once in blue and once in red. Participant had to indicate the color of the item (e.g., press left for a red item, press right for a blue item or vice versa) as quickly and accurately as possible, but only if the item belonged to the memorized sequence. During the control phase at the end of each sequence, three pairs of items were successively presented on the screen. For each couple of items, participants had to indicate whether the first item of the pair was presented before or after the second item of the pair in the memorized sequence. Participants had to press on the letter "o" if the first item was presented before the second and on the letter " $n$ " if this was not the case. The items presented in each pair were always next to each other in the memorized sequence. The entire block was introduced again at the end of the experiment if the participant responded erroneously to at least one of the three pairs. Each participant performed both conditions (in different sessions) and the order of conditions was counterbalanced across participants.

\section{Results and Discussion}

Across both conditions (verbal and spatial), four participants were excluded from the analysis. Two participants-one in the verbal condition and the other in the spatial condition-made too many errors (more than $2 S D$ s above the mean of errors) whereas two other participants - one in the verbal condition and the other in the spatial condition-responded too slowly (more than 2 SDs above the mean of the RTs).

Verbal condition. It took on average 18.47 blocks ( $S D=$ 4.73) before all 16 blocks were correctly performed. During the classification phase, average RT was $827.57 \mathrm{~ms}(S D=101.62 \mathrm{~ms})$ and the average number of errors was $4.97 \%(S D=2.32 \%)$.

The WM Position $\times$ Response Side $(4 \times 2)$ repeated measures AVOVA indicated a main effect of WM position, $F(3,87)=$ 12.24, $p<.001, \eta_{P}^{2}=.30$. Average RTs per position increased gradually $(799,821,840$, and $851 \mathrm{~ms}$ for each position, respectively). A polynomial contrast confirmed the linear trend of these RTs, $F(1,29)=28.70, p<.001, \eta_{P}^{2}=.25$, suggesting that the serial order of the items was processed. A significant interaction between $\mathrm{WM}$ position and response side was observed, $F(3,87)=$ $3.15, p<.05, \eta_{P}^{2}=.10$, indicating an ordinal position effect. The results of the regression analysis were in accord with the ANOVA, $\mathrm{dRT}=-13.95 \mathrm{~ms}, t(29)=-2.26, p<.05$ (Figure 1E). A BF of 1.73 was observed with the Bayesian independent samples $t$ test on verbal regression slopes. This result provides anecdotal evidence for the presence of an ordinal position effect with verbal material.

Spatial condition. It took on average 19.87 blocks $(S D=$ 2.44) before all 16 blocks were correctly performed. During the classification phase, average RT was $683.52 \mathrm{~ms}(S D=88.33 \mathrm{~ms})$ and the average number of errors was $3.31 \%(S D=2.07 \%)$.

The WM Position $\times$ Response Side $(4 \times 2)$ repeated measures ANOVA indicated a significant main effect of WM position, $F(3$, $87)=12.50, p<.001, \eta_{P}^{2}=.30$. Average RTs per position increased gradually $(671,673,677$, and $713 \mathrm{~ms}$ for each position, respectively). A polynomial contrast confirmed the linear trend of these RTs, $F(1,29)=20.63, p<.001, \eta_{P}^{2}=.58$. In line with Experiments 1 and 2, the interaction between WM position and response side was not significant, $F(3,87)=.63, p=.60, \eta_{P}^{2}=$ .021 . This was confirmed by the regression analysis also failing to confirm the presence of the ordinal position effect, slope $=-0.62$; $t(29)=-0.13, p=.90$ (Figure $1 \mathrm{~F}$ ). The Bayesian independent samples $t$ test on the spatial regression slopes resulted in a BF of 0.12 , providing substantial evidence for the absence of an ordinal position effect with spatial material.

Finally, the difference between the linear trends for the verbal and the spatial condition failed to reach significance, even though a more negative slope was observed for the verbal compared to the spatial condition, -13.95 versus $-0.62 ; t(29)=-1.77, p=.086$. This was confirmed with the Bayesian paired samples $t$ test revealing a $\mathrm{BF}$ of 0.78 suggesting anecdotal evidence for an absence of difference in ordinal position effect between the verbal and the spatial condition.

Concerning the presence or the absence of the ordinal position effect, a similar pattern of results was obtained across the three experiments. For all verbal and spatial conditions, both inferential and Bayesian statistics largely agreed, providing evidence for an ordinal position effect with verbal information but not with spatial information. However, across experiments, the interpretation of the results for the direct comparison between the verbal and the spatial conditions is less straightforward. Strong evidence for a difference was observed in Experiment 2, anecdotal evidence was observed in Experiment 1 while no evidence was observed in Experiment 3. To investigate this more closely, a final analysis was conducted investigating the difference between the verbal and the spatial condition across the three experiments, and this was done using both inferential and Bayesian statistics.

A repeated measures ANOVA with condition (2: verbal, spatial) as within-subjects factor and experiment (3: Experiment 1,2 and 3) as between-subjects factor was conducted on the regression slopes. The main effect of condition was significant, $F(1,89)=15.38$, $p<.001, \eta_{P}^{2}=.15$, indicating a dissociation between the spatial and verbal slopes. A separate one-sample $t$ test on these combined regression slopes revealed an ordinal position effect in the verbal condition, $\mathrm{dRT}=-15.73 \mathrm{~ms}, t(91)=-4.82, p<.001$, but not in the spatial condition, $\mathrm{dRT}=1.22 \mathrm{~ms}, t(91)=0.40, p=.69$. Importantly, the interaction between condition and experiments was far from significant ( $p=.82$ ) suggesting that the verbal and the spatial condition behaved similarly across the three experiments. These results were in full agreement with a subsequently run $\mathrm{BF}$ model comparison on the same regression values. In line with the inferential analyses, the best model was the model including only the main effect of condition. This model was preferred by a factor of 8.45 compared to the second model including the main effect of condition and the interaction Experiment-condition, providing substantial evidence for an absence of this interaction. In sum, a comparison across all three experiments using both inferential and Bayesian statistics all converged on the same conclusion. An ordinal position effect in the verbal condition, the absence of the ordinal position effect in the spatial condition, and the difference between both conditions being similar across the three experiments. 


\section{Experiment 4}

Across Experiments 1, 2, and 3, a clear dissociation was observed when classifying verbal or spatial information in WM: An ordinal position effect was observed in the verbal condition while this was not the case in the spatial condition. A straightforward conclusion could be that serial order is coded in a different fashion in verbal and spatial WM, thereby supporting the idea that serial order is not coded in a domain general fashion. Importantly however, it can also be argued that the lack of an ordinal positon effect in the spatial condition is due to the spatial nature of memoranda, which could have interfered with the ordinal position effect itself (e.g., attention based rehearsal, Awh, Jonides, \& Reuter-Lorenz, 1998). If this is the case, the ordinal position effect should not be bound to verbal information, but should also be observed with visual information that does not explicitly contain spatial information. In the current experiment, we further investigate the idea that serial order in WM is only coded spatially when information is used that can be verbalized. This hypothesis is investigated using a cross-sectional experiment with visual information containing a condition with abstract drawings (difficult to verbalize) and a condition with concrete drawings (easy to verbalize). Importantly, each subject participated in both conditions and was asked afterward which strategy he or she had used to maintain the sequence in WM. We expected that all subjects in the concrete condition would use a verbal strategy, whereas in the abstract condition we expected that only a minority of the subjects would do so. By dividing the subjects in terms of the use of a verbal strategy (or not) in the abstract condition, it is possible to determine the importance of verbalization for the ordinal position effect to occur.

\section{Participants}

In total, 51 new undergraduate students from the Université Libre de Bruxelles received course credits to participate in the study. They were all at least 18 years old (on average 21.51 years $(S D=2.76))$. There were 40 females (six left-handed) and 11 males (one left-handed).

\section{Material}

Two different sets of stimuli were constructed to serve for the concrete and the abstract conditions. The concrete set consisted of 64 drawings in black and white colors of objects and food from two drawing databases (Alario \& Ferrand, 1999; Bonin, Peereman, Malardier, Méot, \& Chalard, 2003). They were selected based on their high frequency and high level of recognition (e.g., drawings representing a "book" or a "ring"). The abstract set consisted of 64 drawings in black and white colors from the BEM 144 memory test (Signoret, 1991). These abstract drawings were designed to be as less nameable as possible. See the Appendix for an illustration of those concrete and abstract images.

\section{Stimuli and Procedure}

The procedure for the abstract and concrete condition was similar to Experiment 3 with the exception that the spatial dots and the words were replaced by abstract and concrete drawings. Each participant performed both conditions (in different sessions) and the order of conditions was counterbalanced within participants. At the end of each condition, participants were asked which strategy they had used to encode and maintain the to-be-remembered items in WM and whether they used the same strategy consistently along the entire condition or not. The exact question was, "What strategy did you use to maintain/encode the sequence in WM?". Participants were free in their wordings to answer the question. Answers like "I assigned a name to these abstract drawings" or "These abstract drawings reminded me of a familiar object, so I used a verbal strategy to maintain the stimuli in memory" were classified as verbal semantic strategy group. Answers like "I maintained the shape and the main features of these drawings during the classification phase" or "I maintained the stimuli visually without using a verbal strategy" were classified in the nonverbalizers group.

\section{Results and Discussion}

Across both conditions (concrete and abstract), five participants were excluded from the analysis. Three of them made too many errors-two in the concrete condition and one in the abstract condition (more than $2 S D$ s above the mean of errors) - and two others (in the concrete condition) responded too slowly (more than 2 SDs above the mean of the RTs).

Abstract drawing condition. Out of the 46 included participants, 17 reported to have consistently verbalized the abstract images during the task while 29 participants indicated not to have used such a strategy at all. For participants using the verbal semantic strategy, it took on average 20.33 blocks $(S D=3.44)$ before all 16 blocks were correctly performed. Their average RT was $913 \mathrm{~ms}(S D=70 \mathrm{~ms})$ and accuracy $94.53 \%(S D=2.90 \%)$. The group nonverbalizers needed on average 20.93 blocks $(S D=$ 3.93 ) before all 16 blocks were correctly performed. Their average RT was $873 \mathrm{~ms}(S D=71 \mathrm{~ms})$ and accuracy $94.73 \%(S D=$ $2.78 \%)$.

To analyze the impact of verbalization on the ordinal position effect, a WM Position $\times$ Response Side $(4 \times 2)$ repeated measures ANOVA was performed with Strategy (2: verbal semantic strategy, no verbalization) as between-subjects factor. This analysis revealed a significant main effect of response side, $F(1,44)=$ 4.17, $p<.05, \eta_{P}^{2}=.09$, indicating that participants responded faster with their right-hand side $(887 \mathrm{~ms})$ than with their left-hand side $(899 \mathrm{~ms})$. The main effect for WM position did not reach significance. The interaction between WM position and response side was significant, $F(1,132)=6.67, p<.001, \eta_{P}^{2}=.13$. Importantly, the three-way interaction between Strategy, WM position and response side was significant, $F(3,132)=3.37, p<.05$, $\eta_{P}^{2}=.07$, indicating that the ordinal position effect was influenced by the strategy used. Confirming the hypothesis that the ordinal position effect depends on the use of a verbal semantic strategy, planned comparisons revealed a significant ordinal position effect for the group who verbalized the WM information, $F(1,44)=$ $8.40, p<.01$, but not for the group who did not verbalize, $F(1$, $44)=.10, p=.32$. Regression analyses confirmed these results (see Figures 2A and 2C): dRTs significantly decreased per position by $27.50 \mathrm{~ms}, t(16)=-2.98, p<.001$, for participants who verbalized whereas no such decrease was observed for participants who did not, $7.13 \mathrm{~ms} ; t(28)=-1.08, p=.290$. In agreement with the inferential analyses, a BF of 5.93 was observed providing substantial evidence that an ordinal position effect was observed in participants who verbalized the abstract drawings. In contrast, a 


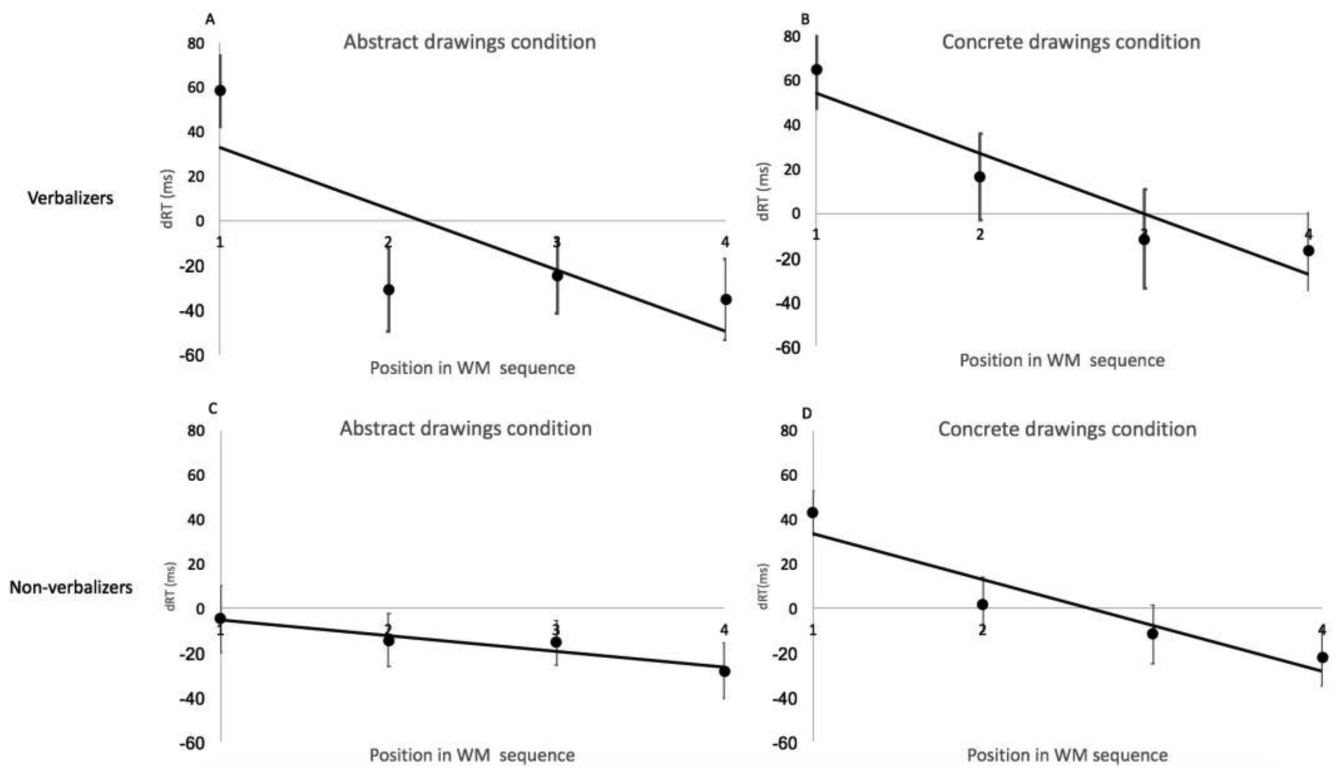

Figure 2. The ordinal position effect with concrete and abstract drawings. Panel A and B depict the results of the abstract and concrete condition of Experiment 3 for the participants who used a verbal strategy in the abstract condition. Panel C and D depict the results of the abstract and concrete condition of Experiment 3 for the participants who did not use a verbal strategy in the abstract condition. The Y-axis represents difference in reaction times (dRTs). These are obtained by subtracting the average RTs from the right hand with the average RT's from the left hand. Each point reflects the dRT for a position in the working memory (WM) sequence. If a dRT has a positive value, left-hand responses are faster than right-hand responses. The black line is obtained after regressing the $\mathrm{dRT}$ to the positions in the sequence. A line with a negative slope is indicative for the expected relation between serial order in WM and space.

BF of 0.33 provided with substantial evidence that the ordinal position effect was not observed in participants who did not verbalize the abstract drawings. Finally, the difference for these regression slopes between both groups just failed to reach significance, -27.50 versus $-7.13 ; t(44)=-1.83, p=.054$. A Bayesian paired samples $t$ test was conducted. The BF of 2.13 provided anecdotal evidence for a difference in regression slope between both groups.

Concrete drawings condition. To ensure that the dissociation reported in the abstract condition is really due to differences in the use of a verbalization strategy and not to other group differences, the data of the concrete condition was analyzed to show that both groups show an indistinguishable ordinal position effect when using a verbalization strategy. Importantly, all participants confirmed having consistently used a verbalization strategy in this condition.

It took on average 18.43 blocks $(S D=3.44)$ before all 16 blocks were correctly performed. During the classification phase, average RT was $831.53 \mathrm{~ms}(S D=74.74 \mathrm{~ms})$ and the average number of errors was $4.72 \%(S D=2.12 \%)$.

The WM Position $\times$ Response Side $\times$ Group $(4 \times 2 \times 2)$ repeated measures ANOVA revealed a main effect of WM position, $F(3,132)=8.00, p<.001, \eta_{P}^{2}=.15{ }^{2}$ Average RTs per position indicated a gradual increase $(823,829,835,850 \mathrm{~ms}$ for each position, respectively). A polynomial contrast confirmed the linear trend of these RTs, $F(1,44)=18.17, p<.001, \eta_{P}^{2}=.29$. The interaction between WM position and response side was also significant $\left[F(3,132)=9.84, p<.001, \eta_{P}^{2}=.18\right]$. Importantly, the three-way interaction between WM position, response side, and group failed to reach significance, $F(3,132)=.22, p=.88$, $\eta_{P}^{2}<.01$, suggesting that the ordinal position effect did not differ in both groups. Regressions analyses confirmed these results (see Figures 2B and 2D): dRTs decreased per position with $27.24 \mathrm{~ms}$, $t(16)=-4.43, p<.001$, for participants who verbalized in the abstract condition and $20.77 \mathrm{~ms}, t(28)=-3.57, p<.002$, for participants who did not verbalize. A BF of 68.21 (verbalizing group) and 26.19 (nonverbalizing group) provided (very) strong evidence supporting the presence of an ordinal position effect in both groups. Finally, these linear trends did not differ, $t(44)=$ $0.72, p=.48$. This was confirmed with a $\mathrm{BF}$ of 0.37 providing anecdotal evidence for the null hypothesis.

\section{Interim Discussion}

In Experiments 1, 2, and 3, a clear dissociation was observed when classifying verbal or spatial information in WM: The activation of spatial position markers was observed in the verbal

\footnotetext{
${ }^{2}$ A separate ANOVA across both conditions (concrete or abstract drawings) did not result in a significant four-way interaction between condition, strategy, WM position, and response side, $F(3,132)=1.305, p=.28$, $\eta_{P}^{2}=.03$. This is not so surprising given that the choice of strategy was expected to vary only in the abstract drawings condition. More specifically, an ordinal position effect was expected in both groups for the concrete drawings and for the verbalizers with the abstract drawings. An absence of ordinal position effect was only expected for the nonverbalizers in the abstract drawings condition.
} 
condition while this was not the case in the spatial condition. To rule out the idea that the absence of the ordinal position effect is due to spatial "noise" induced when maintaining spatial information, we directly investigated in Experiment 4 whether the ordinal position effect depends on the verbalisation of the stimuli or not. Instead of presenting words or locations, drawings of abstract (difficult to verbalize) and concrete (easy to verbalize) stimuli were presented to the same participants. The results demonstrated that, regardless of whether the stimuli were abstract or concrete, the ordinal position effect was only observed if participants reported having used a verbal strategy. This finding supports the idea that serial order coding in verbal and visuospatial WM draws upon dissociable mechanisms. At the same time, they highlight the importance to carefully control verbalization strategies when studying modality specificity of certain cognitive functions.

To obtain further insights in the role of verbalization in the spatial coding of serial order in WM, we conducted a fifth experiment using an articulatory suppression task. This type of task involves that the articulation of irrelevant verbal information during the task prevents the rehearsal of the verbal material to memorize (Baddeley, 2007, 2010; Baddeley \& Logie, 1999). This implies that if verbalization is essential to observe an ordinal position effect, it should disappear under articulatory suppression.

\section{Experiment 5}

In Experiment 4, a relation was observed between the occurrence of the ordinal position effect and the participants' report of having used a verbal strategy to process the stimuli in WM or not. Here, in Experiment 5 the specific role of verbalization is investigated. Participants again encode and classify the concrete drawings used in the previous experiment. This time, however, participants concurrently perform an articulatory suppression task or a foot tapping task. This concurrent articulatory task would prevent the verbalization of the concrete drawings and should lead to a decrease or absence of the ordinal position effect. To maximize the chances of observing an influence of articulatory suppression, two separate experiments were conducted with participants performing the secondary suppression task concurrently with either the encoding or the classification phase.

\section{Participants}

In total, 61 new undergraduate students from the Université Libre de Bruxelles received course credits to participate in the experiment They were all at least 18 years old (on average 22.60 years, $S D=2.99$ ). Forty-six participants were female (two lefthanded) and 15 were male (three left-handed)]. Twenty-eight of them (on average 22.55 years, $S D=2.76 ; 21$ females, all righthanded, and seven males, one left-handed) were tested in the suppression during encoding study. The other 33 participants (on average 22.64 years, $S D=3.22 ; 25$ females, two left-handed, and eight males, two left-handed) were tested in the suppression during classification study. Participants performed three sessions of 30 min each, on three consecutive days.

\section{Material, Stimuli, and Procedure}

Participants performed exactly the same task as in the previous experiments but in three different conditions: with concurrent articulatory suppression, with concurrent foot tapping, and without any secondary task (i.e., control condition). The foot tapping condition was added to ensure that the results observed in the articulatory suppression condition would not be due to the additional cognitive load of dual task. Each participant was randomly assigned to one of two studies (either suppression during encoding or suppression during classification) and performed the three conditions (in different sessions). The order in which participants completed the classification of the concrete drawings for the three conditions was counterbalanced across participants.

At the beginning of the foot tapping and articulatory suppression conditions, the experimenter first described the secondary task and demonstrated how to perform it. Participants were instructed to say /patipato/ aloud and repeatedly once per second in the articulatory suppression condition, or to tap a foot once per second in the foot tapping condition. After receiving the instructions, participants practiced the secondary task to make sure that the task requirements were clear and that they could perform the task correctly. In the suppression during encoding study, participants were asked to perform the concurrent secondary task during the encoding phase of the main task. In the suppression during classification study, participants were instructed to perform the concurrent secondary task during the classification phase of the main task. Performance in the foot tapping and articulatory suppression task was closely monitored by the experimenter.

\section{Results and Discussion}

Experiment 5a: Suppression during encoding. Across the three conditions (control, articulatory suppression and foot tapping), four participants were excluded from the analysis. Three of them made too many errors-one in each condition (more than 2 $S D$ s above the mean of errors) - and another responded too slowly (more than 2 SDs above the mean of the RTs) in all conditions.

Control condition. In this condition, it took on average 16.87 blocks $(S D=0.99)$ before all 16 blocks were correctly performed. During the classification phase, average RT was $802.94 \mathrm{~ms}(S D=$ $66.96 \mathrm{~ms})$ and the average number of errors was $4.01 \%(S D=$ $1.99 \%)$.

The WM Position $\times$ Response Side $(4 \times 2)$ repeated measures ANOVA indicated that the effect of WM position just failed to reach significance, $F(3,69)=2.67, p=.054, \eta_{P}^{2}=.10$. Average RTs per position were $798,794,818$, and $813 \mathrm{~ms}$ and a polynomial contrast confirmed the linear trend of these RTs, $F(1,23)=5.88$, $p<.05, \eta_{P}^{2}=.20$. An interaction between $\mathrm{WM}$ position and response side was observed, $F(3,69)=4.82, p<.005, \eta_{P}^{2}=.17$, indicating position-space associations. The regression analysis confirmed the presence of an ordinal position effect with the difference between right- and left-hand responses decreasing with $22.33 \mathrm{~ms}$ per position, $\mathrm{dRT}=-22.33 \mathrm{~ms}, t(23)=-3.50, p<.005$ (Figure 3A). A Bayesian independent samples $t$ test resulted in a $\mathrm{BF}$ of 19.63 , providing strong evidence for the presence of an ordinal position effect.

Foot tapping condition. It took, on average, 18.46 blocks $(S D=3.22)$ before all 16 blocks were correctly performed. During the classification phase, average RT was $827.17 \mathrm{~ms}(S D=100.12$ $\mathrm{ms})$ and the average number of errors was $4.67 \%(S D=2.89 \%)$.

The WM Position $\times$ Response Side $(4 \times 2)$ repeated measures ANOVA revealed a main effect of $\mathrm{WM}$ position, $F(3,69)=5.32$, 

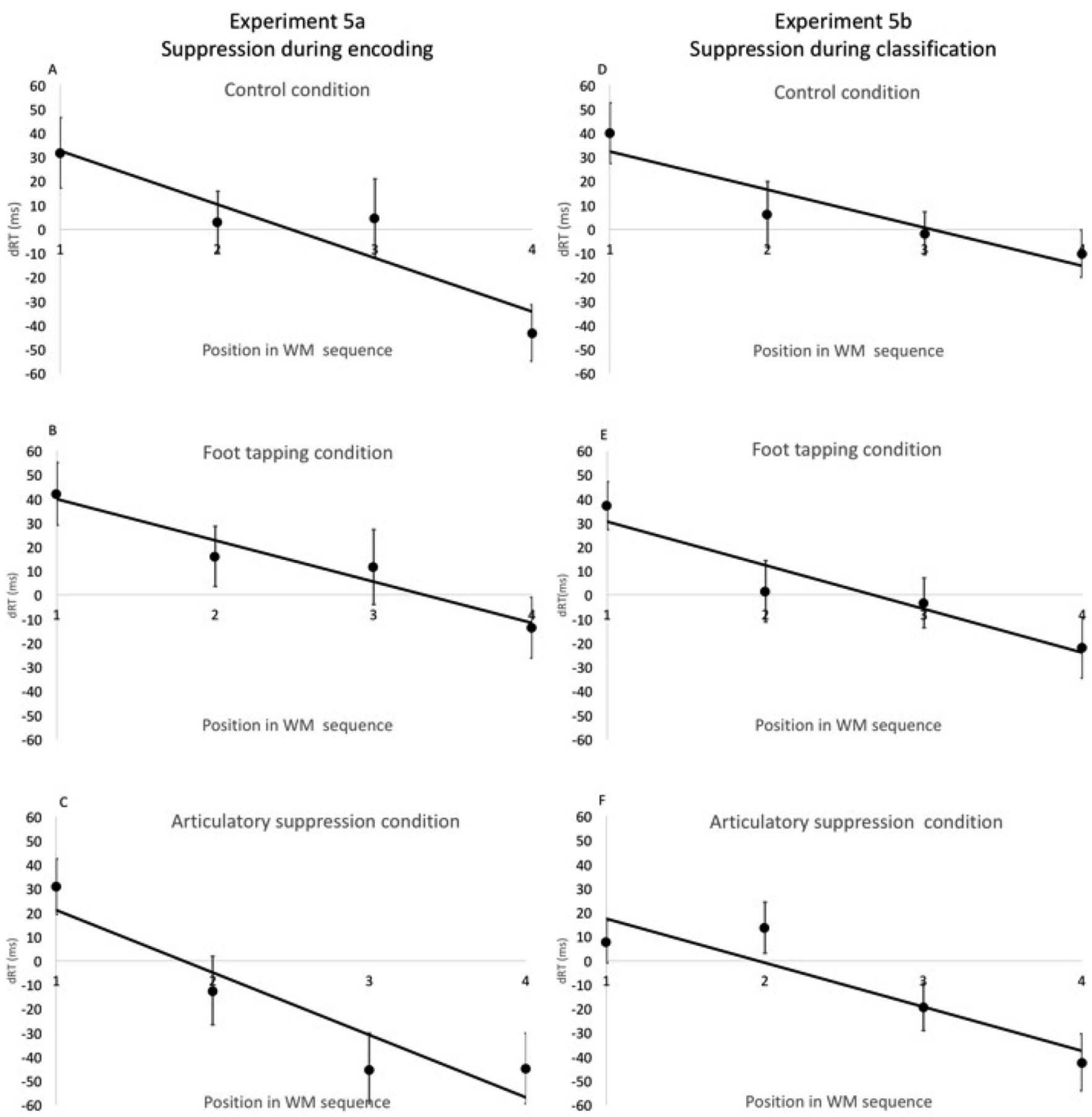

Figure 3. The ordinal position effect of Experiment 5. Panels A, B, and C depict the results of the control, foot tapping and articulatory suppression condition of suppression during encoding study. Panels D, E, and F depict the results of the control, foot tapping and articulatory suppression condition of suppression during classification study. The Y-axis represents difference in reaction times (dRTs). These are obtained by subtracting the average RTs from the right hand with the average RT's from the left hand. Each point reflects the dRT for a position in the working memory (WM) sequence. If a dRT has a positive value, left-hand responses are faster than right-hand responses. The black line is obtained after regressing the dRT to the positions in the sequence. A line with a negative slope is indicative for the expected relation between serial order in WM and space.

$p<.005, \eta_{P}^{2}=.19$. Average RTs per position indicated a gradual increase $(805,821,830$, and $845 \mathrm{~ms}$ for each position, respectively). A polynomial contrast confirmed the linear trend of these RTs, $F(1,23)=15.13, p<.001, \eta_{P}^{2}=.40$. The interaction between WM position and response side was again significant, $F(3,69)=3.51, p<.05, \eta_{P}^{2}=.13$. The regression analysis support the conclusion of the ANOVA, dRT $=-17.15 \mathrm{~ms}$, $t(23)=-3.07, p<.005$ (Figure 3B). Finally, a Bayesian independent samples $t$ test resulted in a BF of 8.17 , providing substantial evidence for the presence of the ordinal position effect.
Articulatory suppression condition. It took on average 21.21 blocks $(S D=4.98)$ before all 16 blocks were correctly performed. During the classification phase, average RT was $857.30 \mathrm{~ms}(S D=$ $89.39 \mathrm{~ms})$ and the average number of errors was $6.07 \%(S D=$ $2.93 \%)$.

The repeated measures ANOVA with WM position and response side as within-subjects factors showed a main effect of WM position, $F(3,69)=3.49, p<.05, \eta_{P}^{2}=.13$. Average RTs per position were $849,840,874$, and $862 \mathrm{~ms}$. The polynomial contrast, highlighting the linear trend of these RTs, just failed to reach 
significance, $F(1,23)=3.14, p=.09, \eta_{P}^{2}=.12$. As in control and foot tapping conditions, an ordinal position effect was observed, indicated by a significant interaction between WM position and response side, $F(3,63)=6.67, p<.001, \eta_{P}^{2}=.23$. The results of the regression analysis were in agreement with the ANOVA. The ordinal position effect is significantly present, dRT $=-25.99 \mathrm{~ms}$, $t(23)=-4.01, p<.001$ (Figure 3C). Finally, a Bayesian independent samples $t$ test resulted in a BF of 58.63, providing very strong evidence for the presence of the ordinal position effect.

A combined analysis was conducted to directly compare the size of the ordinal position effect between the three conditions. In the repeated-measures ANOVA including the ordinal position in WM (4: 1 to 4), response side (2: left, right) and condition (3: control, foot tapping, articulatory suppression) as within-subjects factors, the factor "condition" did not interact with the ordinal position effect $(p=.39)$ nor with the factor "position" $(p=.23)$. The main effect of condition was significant, $F(2,46)=5.316, p<.01$, $\eta_{P}^{2}=.19$. Planned comparisons revealed that average RT was significantly longer for the articulatory suppression condition compared to the control condition ( $856.58 \mathrm{~ms}$ vs. $805.88 \mathrm{~ms}), F(1$, $23)=8.529, p<.01, \eta_{P}^{2}=.27$, as well as the foot tapping condition (856.58 ms versus $825.18 \mathrm{~ms}), F(1,23)=4.623, p<$ $.05, \eta_{P}^{2}=.17$. In addition, participants performed significantly more blocks in the articulatory suppression condition before all 16 blocks were correctly completed compared to the control condition (21.21 blocks versus 16.87 blocks), $F(1,23)=18.016, p<.001$, $\eta_{P}^{2}=.44$, as well as the foot tapping condition (21.21 blocks versus 18.46 blocks), $F(1,23)=6.301, p<.05, \eta_{P}^{2}=.21$. The difference between the control and the foot tapping conditions was also significant (16.87 blocks versus 18.46 blocks), $F(1,23)=$ 5.128, $p<.05, \eta_{P}^{2}=.18$. In sum, RTs and performance suggest that the suppression tasks were correctly performed. At the same time, there is no influence observed of articulatory suppression on the ordinal position effect.

Experiment 5b: Suppression during classification. Across the three conditions (control, articulatory suppression and foot tapping), five participants (one in the control condition, two in the foot tapping condition and two in the articulatory suppression condition) were excluded from the analysis because they made too many errors (more than $2 S D$ s above the mean of errors).

Control condition. In this condition, it took on average 17.21 blocks $(S D=1.75)$ before all 16 blocks were correctly performed. During the classification phase, average RT was $815.64 \mathrm{~ms}(S D=$ $95.82 \mathrm{~ms})$ and the average number of errors was $3.56 \%(S D=$ $1.74 \%)$.

The WM Position $\times$ Response Side $(4 \times 2)$ repeated measures ANOVA did not show a significant main effect of WM position $(p=.34)$. There was a significant interaction between WM position and response, $F(3,81)=3.66, p<.05, \eta_{P}^{2}=.12$. The regression analysis confirmed the presence of an ordinal position effect with the difference between right- and left-hand responses decreasing with $15.85 \mathrm{~ms}$ per position, dRT $=-15.85 \mathrm{~ms}$, $t(27)=-3.65, p<.001$ (Figure 3D). Finally, a Bayesian independent samples $t$ test resulted in a BF of 30.94, providing very strong evidence for the presence of the ordinal position effect.

Foot tapping condition. It took, on average, 17.82 blocks $(S D=2.60)$ before all 16 blocks were correctly performed. During the classification phase, average RT was $823.80 \mathrm{~ms}(S D=88.43$ $\mathrm{ms})$ and the average number of errors was $4.02 \%(S D=1.51 \%)$.
The WM Position $\times$ Response Side $(4 \times 2)$ repeated measures ANOVA did not show a significant main effect of WM position $(p=.26)$. The interaction between WM position and response side was significant, $F(3,81)=5.29, p<.05, \eta_{P}^{2}=.16$. The regression analyses support the conclusion of the ANOVA (dRT $=-18.18 \mathrm{~ms})$, $t(27)=-3.07, p<.005$ (Figure 3E). Finally, a Bayesian independent samples $t$ test resulted in a BF of 22.16, providing strong evidence for the presence of the ordinal position effect.

Articulatory suppression condition. It took on average 19.28 blocks ( $S D=3.70$ ) before all 16 blocks were correctly performed. During the classification phase, average RT was $818.59 \mathrm{~ms}(S D=$ $66.74 \mathrm{~ms})$ and the average number of errors was $4.73 \%(S D=$ $2.04 \%)$

The $4 \times 2$ (WM Position $\times$ Response Side) repeated measures ANOVA did not show a significant main effect of WM position $(p=.24)$. As in control and foot tapping conditions, an ordinal position effect was observed, indicated by a significant interaction between WM position and response side, $F(3,81)=6.89, p<$ $.001, \eta_{P}^{2}=.20$. The results of the regression analysis were in accord with the ANOVA. The ordinal position effect is significantly present $(\mathrm{dRT}=-18.33 \mathrm{~ms}), t(27)=-4.29, p<.001$ (Figure 3F). A Bayesian independent samples $t$ test resulted in a $\mathrm{BF}$ of 8.17 , providing substantial evidence for the presence of the ordinal position effect.

A second repeated-measures ANOVA was run with ordinal position in WM (4: 1 to 4), response side (2: left, right) and condition (3: control, foot tapping, articulatory suppression) as within-subjects factors to directly compare the size of the ordinal position effect between the 3 conditions. The three-way interaction between WM position, response side and condition was not significant ( $p=.37$ ) nor was the interaction between WM position and condition $(p=.65)$. The main effect of condition was not significant $(p=.85)$. Finally, participants performed more blocks in the articulatory suppression condition before all 16 blocks were correctly completed compared to the control condition (19.28 blocks versus 17.21 blocks), $F(1$, $27)=8.236, p<.01, \eta_{P}^{2}=.23$, as well as compared to the foot tapping condition (19.28 blocks versus 17.82 blocks), $F(1$, 27) $=6.727, p<.05, \eta_{P}^{2}=.20$.

Finally, we ran a repeated-measures ANOVA with ordinal position in WM (4: 1 to 4), response side (2: left, right) and condition (3: control, foot tapping, articulatory suppression) as withinsubjects factors and study (2: suppression during encoding, suppression during classification) as between-subjects factor. The factor "study" did not interact significantly with our effects of interest.

Concurrent tasks had an influence on general performance. Dual task performance resulted in the need for more blocks to complete the task. This was the case both during encoding and during classification. Furthermore, with suppression during encoding, participants responded slower in the articulatory suppression condition compared to the two other conditions. Together, these results suggest that participants correctly performed the dual task conditions and that the suppression had an influence on task performance. Surprisingly, articulatory suppression had absolutely no effect on the ordinal position effect. These results suggest that verbalization is not playing a key role in the creation of spatial codes when categorizing concrete drawings in WM. 


\section{Experiment 6}

At first sight, the observations made in Experiment 4 and 5 seem contradictory. In Experiment 4 it was observed that the creation of spatial associations when categorizing abstract drawings depended on whether participants did or did not verbalize these drawings. In Experiment 5, on the other hand, spatial associations were created when participants classified concrete drawings, regardless of verbalization. Combining the results of the two previous experiments, it seems that verbalization of memorized information is crucial for abstract (Experiment 4), but not for concrete (Experiment 5) drawings. How can these seemingly disparate findings be reconciled? Recently, some authors argued that the processing of order information is influenced by semantic representation of information within a long-term memory network (Acheson, MacDonald, \& Postle, 2011; Poirier, Saint-Aubain, Mair, Tehan, \& Tolan, 2015). The question asked in Experiment 6 is whether the semantic representation, rather than the verbalization of information, can be crucial for the creation of spatial associations of order information in WM.

A short overview of the previous experiments reveals that semantic representation could indeed be an important variable. In Experiments 1 to 3 spatial associations were observed when words were used referring to fruits and vegetables (Experiments 1 and 2) or to words referring to other objects (Experiment 3). Such words are automatically verbalized and it is impossible not to activate the semantic content of these words upon reading (Stroop, 1935). Instead no spatial associations were observed with spatial positions. In Experiment 4 spatial associations were observed with abstract drawings, but only if these drawings were assigned a semantic representation (i.e., were verbalized). Finally, it is interesting to note that verbalization of concrete drawings was prevented using articulatory suppression in Experiment 5, their semantic content was still preserved (Poirier et al., 2015).

In the following experiment we will directly investigate the importance of semantic representation of the memorized information to get spatial coding of serial order. To do so, participants performed both a condition with pseudowords (without meaning) and a condition with words referring to existing objects (with meaning). We deliberately chose verbal material (words and pseudowords) to be sure that participants always verbalized the stimuli. Moreover, given that presenting pseudowords can activate word representation in LTM (e.g., McClelland \& Rumelhart, 1981), we assumed that participants had room to associate semantic content to the pseudowords they had to learn. To measure the frequency with which this happened, each participant was asked to provide with an estimate of the proportion of trials where he or she assigned a semantic representation to the pseudowords. If the activation of semantic information assigned to the items drives the activation of spatial position markers, we predicted that the proportion of trials where a semantic content was assigned to pseudowords would relate to the size of the ordinal position effect.

\section{Participants}

Eighty-two new undergraduate students ${ }^{3}$ from the Université Libre de Bruxelles received course credits to participate in the study. They were all at least 18 years old (on average 20.34 years,
$S D=3.08)$. There were 68 females (three left-handed) and 14 males (two left-handed).

\section{Material}

The material used for the words condition was identical to the verbal condition of Experiment 3 (i.e., a set of 64 words referring to 64 concrete drawings that have been used in Experiment 4). A matched set of 64 pseudowords was created using the multilingual pseudoword generator Wuggy (Keuleers \& Brysbaert, 2010). The set of words and pseudowords was matched on number of letters and number of syllables, as well as transition frequencies and subsyllabic structure.

\section{Stimuli and Procedure}

Experiment 6 followed exactly the same procedure as Experiments 3 and 4 and differed only in the type of stimuli that were used (i.e., words and pseudowords). Each participant performed both conditions (in different sessions) and the order of conditions was counterbalanced across participants. At the end of the pseudowords condition, participants received a questionnaire to investigate whether they had assigned a meaning to the pseudowords or not. The exact question was "How did you encode/maintain the items in WM?" and predetermined answers were provided to the participants. These were "I encoded/maintained the pseudo-words as presented," "I encoded/maintained only some letters of pseudowords," "I encoded/maintained known words instead of pseudowords," or "other." Participants were also asked to indicate the proportion (in percentage) with which they used each of these strategies.

\section{Results and Discussion}

Across the two conditions (words and pseudowords), seven participants were excluded from the analysis. Five of them made too many errors (more than 2 SDs above the mean of errors) - four in the words condition and one in the pseudowords conditionone of them responded too slowly (more than 2 SDs above the mean of the RTs) in the words condition and another made too many errors as well as responded too slowly in the pseudowords condition.

Pseudoword condition. It took on average 23.48 blocks ( $S D=4.49$ ) before all 16 blocks were correctly performed. During the classification phase, average RT was $929.60 \mathrm{~ms}(S D=82.52$ $\mathrm{ms})$ and the average number of errors was $9.54 \%(S D=4.80 \%)$.

In a first step, a repeated measures analysis of covariance (ANCOVA) with ordinal position in WM (4: 1 to 4), response side (2: left, right) as within-subjects factors and semantic assignment (proportion of semantic content assigned to pseudowords) as a covariate was performed in order to evaluate the influence of semantic representation on the ordinal position effect. This anal-

\footnotetext{
${ }^{3}$ Following a reviewer's comment, we increased the size of the sample compared to previous experiments to ensure the reliability of the effects, but note that the pattern of results presented below was already obtained with 40 participants. To achieve an adequate power of .80 for the correlation between the ordinal position effect and assignment of semantic representation to the pseudowords, the sample required was 75 participants.
} 
ysis revealed a significant main effect of WM position, $F(3$, $219)=3.85, p<.05, \eta_{P}^{2}=.05$. Average RTs per position increased gradually $(914,927,933$, and 939). A polynomial contrast confirmed the linear trend of these RTs, $F(1,73)=9.64, p<$ $.005, \eta_{P}^{2}=.12$. The interaction between ordinal position and response side was not significant, indicating the absence of the ordinal position effect, $F(3,219)=1.97, p=.12, \eta_{P}^{2}=.03$. Importantly, the ordinal position effect interacted with the factor "semantic assignment," $F(3,219)=9.71, p<.001, \eta_{P}^{2}=.12$. This factor did not covary with any other effect.

To further define the impact of semantic assignment on position-space associations, participants were divided into three equal groups according to the proportion of sematic content assigned to pseudowords. More precisely, 25 participants reported not to have assigned a semantic representation to information (proportion of semantic content of 5\% or smaller) were included in "no semantic content" group. Twenty-five participants reported to have infrequently assigned a semantic representation to information (proportion of semantic content from 6\% to 39\%) were included in low semantic content group. Twenty-five participants reported to have frequently assigned a semantic representation to information (proportion of semantic content of $40 \%$ or larger) were included in high semantic content group. The WM Position $\times$ Response Side $(4 \times 2)$ repeated measures ANOVA with level of semantic representation (3; no semantic content, low semantic content, high semantic content) as between-subjects factor revealed a main effect of WM position, $F(3,216)=6.28, p<$ $.001, \eta_{P}^{2}=.08$. The interaction between $\mathrm{WM}$ position and response side was significant, $F(3,216)=9.05, p<.001, \eta_{P}^{2}=.11$. Importantly, the three-way interaction between semantic, WM position and response side was significant, $F(6,216)=3.20, p<$ $.005, \eta_{P}^{2}=.08$, indicating that the ordinal position effect was influenced by the proportion of semantic representation assigned to information. In the "no semantic content" group, the interaction between WM position and response side just failed to reach significance, $F(3,72)=2.62, p=.057, \eta_{P}^{2}=.10$. Closer inspection of this interaction showed that the positions 2 and 3 were responded faster with the right hand while no difference between left and right responses was observed for the Positions 1 and 4 . Therefore, although the reason for this interaction remains unclear, it certainly does not reflect an ordinal position effect (see also the regression analyses). In the low semantic content group, the interaction between WM position and response side failed to reach significance, $F(3,72)=1.29, p=.28, \eta_{P}^{2}=.05$. In the high semantic content group, the interaction between WM and response side was significant, $F(3,72)=10.79, p<.001, \eta_{P}^{2}=.31$, replicating the ordinal position effect. Regression analyses confirmed these results (see Figure 4A): dRTs significantly decreased per position by $38.33 \mathrm{~ms}, t(24)=-5.15, p<.001$, for high semantic content group although no such decrease was observed for no semantic content group $(-2.62 \mathrm{~ms}), t(24)=-0.47, p=$ .64 , nor for the low semantic content group (10.21 ms), $t(24)=-1.61, p=.12$. In full agreement with the inferential analyses, a BF of 0.23 was observed, providing substantial evidence for the absence of an ordinal position effect in no semantic content group. A BF of 0.66 was observed for the low semantic content group. This value, below 1 , suggests anecdotal evidence for the null hypothesis. Finally, a BF of 834.91 was observed in the high semantic content group, providing extreme support for the presence of the ordinal position effect.

Finally, the comparison of regression slopes between the three groups was significant $[F(2,72)=8.39, p<.001]$. Planned comparisons revealed that the slope for the high semantic content group significantly differed from the no semantic content group $(p<.001)$ and from the low semantic content group $(p<.005)$. The comparison of regression slopes between the no semantic content and the low semantic content groups was not significant $(p=.41)$. The same conclusion can be made on the basis of a Bayesian ANOVA on the three groups with regression slopes as dependent measure. This model outperformed the null-model by a factor of 57.6. Direct comparisons yielded anecdotal evidence for an absence of difference between the no semantic content and the low semantic content group $(\mathrm{BF}=0.39)$, whereas substantial $(\mathrm{BF}=7.29)$ and very strong evidence $(\mathrm{BF}=73.0)$ were observed for the comparisons no versus high semantic content and low versus high semantic content, respectively.

Word condition. It took on average 19.17 blocks $(S D=2.79)$ before all 16 blocks were correctly performed. During the classification phase, average RT was $821.30 \mathrm{~ms}(S D=92.98 \mathrm{~ms})$ and the average number of errors was $4.32 \%(S D=3.02 \%)$.
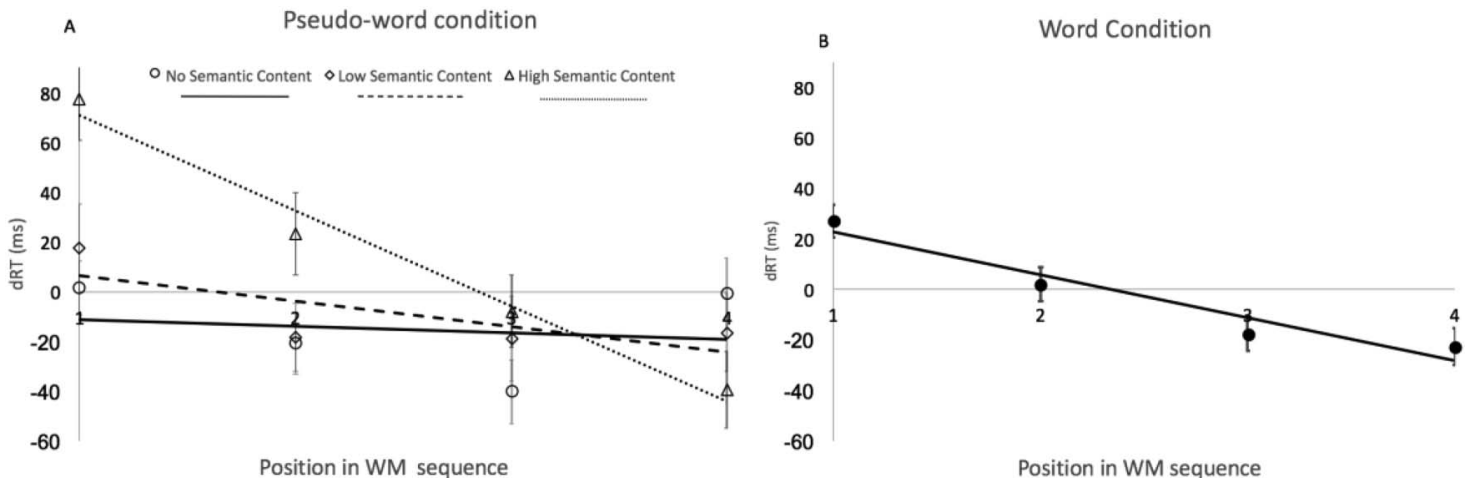

Figure 4. The ordinal position effect with words and pseudowords. Observed data and regression line of Experiment 6, representing RT differences between right and left responses in function of the position in working memory sequence for the words condition (A) and the pseudowords condition (B). Positive values reflect faster left-hand than right-hand responses. 
The WM Position $\times$ Response Side $(4 \times 2)$ repeated measures ANOVA revealed a main effect of WM position, $F(3,222)=$ $14.34, p<.001, \eta_{P}^{2}=.16$. Average RTs per position increased gradually $(807,816,822$, and $840 \mathrm{~ms}$ for each position, respectively). A polynomial contrast confirmed the linear trend of these RTs, $F(1,74)=33.99, p<.001, \eta_{P}^{2}=.31$, indicating that the serial order of the sequence was processed. The interaction between WM position and response side, $F(3,222)=11.53 p<$ .001, $\eta_{P}^{2}=.14$, was also significant. The regression analysis confirmed the presence of the ordinal position effect $(\mathrm{dRT}=-16.91 \mathrm{~ms}), t(74)=-5.36, p<.001$ (Figure 4B). A BF of 16,498.98 was observed, providing extreme evidence for the presence of an ordinal position effect with the Bayesian independent samples $t$ test. For completeness, we also ran the repeated measures ANOVA by adding level of semantic representation (3; no semantic content, low semantic content, high semantic content) as between-subjects factor to ensure that the results obtained in the pseudoword condition is really due to differences in the proportion of semantic content assigned to information and not to other group differences. The level of semantic representation did not interact significantly with the ordinal position effect $(p=.52)$. Moreover, the comparison of slopes between the three groups was not significant $(p=.68)$. These results suggest that the ordinal position effect did not differ between groups in word condition.

Relation between the ordinal position effect and assignment of semantic representation to the pseudowords. To substantiate the claim that the ordinal position effect is related to assignment of semantic content to the stimuli, a correlation was calculated between the size of the ordinal position effect and the proportion of trials in which a semantic representation was assigned to the pseudowords. A negative correlation was observed, $r(75)=-.53, p<.001$, demonstrating that participants with higher proportions of semantic representation assignment also had stronger ordinal position effects (see Figure 5). Finally, a BF of 17,534 was observed for this correlation, providing with extremely strong support, confirming the relation between the ordinal position effect and the assignment of semantic content.

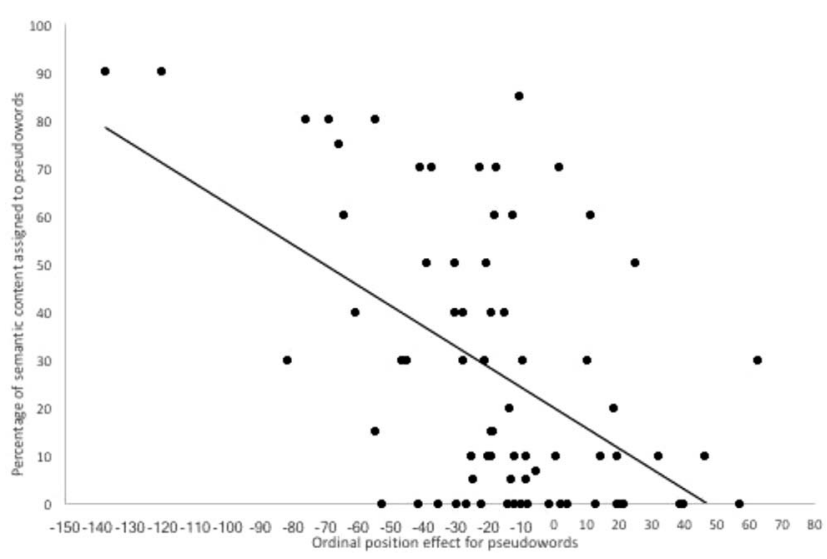

Figure 5. Correlation between proportion of semantic content assigned to information and ordinal position effect. Scatter plot of the correlation between the ordinal position effect for pseudowords (X-axis) and the percentage of meaning assigned to pseudowords (Y-axis). Negative values on the $\mathrm{X}$ axis indicate the presence of the ordinal position effect.

\section{General Discussion}

The aim of the current study was to investigate whether serial order coding in WM works according to the same principles in the verbal and the visuospatial domain. In Experiments 1, 2, and 3, a dissociation was observed when coding ordinal information of a verbal or of a spatial nature. The ordinal position effect was robustly observed with verbal information, while it was repeatedly absent with spatial information. The subsequent experiments investigated the nature of this dissociation. Experiment 4 further explored whether the use of a verbal strategy could be important for the creation of spatial codes when holding ordinal information in WM. In different conditions, abstract (difficult to apply a verbal strategy) and concrete (easy to apply a verbal strategy) visual stimuli were presented to the same participants. Regardless of whether the stimuli were abstract or concrete, the ordinal position effect was observed. Closer inspection revealed that the ordinal position effect was only observed if participants reported having used a verbal strategy to process the visual stimuli in WM. The ordinal position effect was not observed when participants did not use such a strategy. Experiment 5 examined the specific contribution of verbalisation for the creation of spatial position markers in WM. For that, we used a dual task paradigm with articulatory suppression. Surprisingly, articulatory suppression had no effect on the ordinal position effect, not during encoding, nor during classification. Consequently, the verbalization of memorized information seems not essential for associating items in WM with spatial position markers. At this point we noted that although verbalization of memorized information should have been abolished during the articulatory suppression task, their semantic content was still preserved (Poirier et al., 2015). In the verbal conditions of the previous experiments, it was not possible to dissociate verbalization and assignment of semantic representation. In Experiment 6 , we therefore explored the role of semantic coding for the creation of the ordinal position effect. This time, participants performed a condition with pseudowords (lacking semantic content) and a condition with words referring to existing objects (with semantic content). When taking proportion of semantic assignment into account, spatial coding was observed in the word condition but not in the pseudoword condition. Given that pseudowords were used (the same in all participants), it does not seem likely that long-term phonological or lexical representations contributed to this spatial coding of the stimuli. Rather, a closer look of the results within the pseudoword condition revealed that the ordinal position effect could be observed with pseudowords, but only if participants associated these pseudowords with a meaning. Consistently, a correlation was observed between the reported frequency of semantic assignment and the size of the ordinal position effect: A higher proportion of semantic assignments to the pseudowords associated with a stronger ordinal position effect. In sum, the results of this final experiment suggest that the semantic coding of information in WM has an important role in the creation of the ordinal position effect. In what follows, the theoretical implications of these findings as well as some open issues will be discussed.

On the basis of the current findings, we agree with the idea that serial order coding in WM occurs within a spatially defined coordinate system, an idea originally put forward in the mental whiteboard hypothesis (Abrahamse et al., 2014). According to this 
idea, when items need to be maintained and retrieved from WM, new temporary bindings are created between these items and their spatial position in a cognitive coordinate system. As such, a conjunction is created associating items with a spatial position tag so that serial order can be retrieved. Note however, that our observations also constrain the mental whiteboard hypothesis (Abrahamse et al., 2014) in an important way. That is, item-space conjunctions would only be created if the items memorized in WM are semantically coded by the participants.

When participants did not, or only infrequently, assign meaning to the memorized stimuli, the ordinal position effect was not observed. This observation makes it possible to speculate why the ordinal position effect was not observed with spatial information in the first three experiments. We speculate that spatial position markers were not created in the spatial condition of the first three experiments because participants did not assign meaning to the spatially presented dots. A possible alternative interpretation of our results could be that spatial position markers were not created in the spatial conditions because they would interfere with the spatial (attentional) nature of the task itself (e.g., attention based rehearsal; Awh, Jonides, \& Reuter-Lorenz, 1998). Even though this possibility cannot be refuted on the basis of our data, it does not explain why the ordinal position effect is not observed when participants categorized abstract visual information. These stimuli did not involve spatial processing but still the ordinal position effect was not observed when the task was solved in a nonverbal and nonsemantic way. Note also that, even though the ordinal position effect was not observed in the spatial conditions of the first three experiments, participants still showed good performance in memorizing the different spatial positions for later recall. In other words, performance was still good, even though (left-to right organized) spatial position markers were not created. This suggests that the creation of horizontal spatial position markers to encode ordinal information in WM, as suggested by the mental whiteboard hypothesis, is not mandatorily. Whether this is a reflection of the flexibility of the spatial position marking (meaning that other than horizontal coordinates can be used) or an indication that different (nonspatial) routes exist to temporarily store order information in $\mathrm{WM}$ is an issue for future investigation.

Our data are in line with recent findings showing that order information in WM is influenced by semantic information (Acheson et al., 2011; Poirier et al., 2015). Using a dual task paradigm, Acheson and colleagues observed that a secondary task involving semantic processing increased the proportion of order judgment errors for concrete words but did not affect order error proportions for pseudowords. On the basis of these findings it was argued that semantic representations have an effect on serial ordering in WM and order recall relies on the activation of items in a semantic network. Similarly, Poirier and colleagues (2015) recently demonstrated that semantic activation impacted order information coding in verbal WM. According to this theory, order information can be coded thanks to the so-called primacy gradient (e.g., the first item in a to be memorized sequence receives more activation than the second, which in turn receives more activation than the third, and so on; . . . Grossberg, 1978a, 1978b). To make an order judgment it would then be sufficient to compare the activation levels that the items received because of their position within the sequence. Crucially, however, activity resulting from the primacy gradient and activity resulting from semantic network activation would not be independent. Differences in the level of the semantic activation of an item could then interact with the primacy gradient activity inducing ordinal judgment errors.

Our results agree with these studies in the sense that semantic network activation is an important factor for ordinal information processing. Indeed, the ordinal position effect was observed when semantic coding was present but was not observed when semantic coding was absent. On the other hand, under the assumption that the serial search effect can be interpreted as a marker of the primacy gradient (e.g., Hurlstone et al., 2014), the ordinal position effect seems not to be strongly depending on the primacy gradient. Indeed, both the serial search effect and the ordinal position effect did not systematically co-occur in the present data. Participants using a verbal and semantic strategy with the abstract visual stimuli in Experiment 4 as well as participants performing the secondary suppression task during the classification phase in Experiment 5 demonstrated an ordinal position effect but no effect of WM position, while in the spatial conditions of Experiments 1, 2 and 3, no ordinal position effect but a main effect of WM position was observed. A main effect of WM position (and especially its linear increasing trend) is typically considered as an index of serial search in WM (e.g., Sternberg, 1975). It thus seems that not the serial search in itself, but semantic processing of the WM items is needed for the creation of spatial markers that give rise to the ordinal position effect. In other words, as soon as such spatial position markers are created, an ordinal position effect is observed regardless of whether participants use a serial scanning strategy during categorization (Experiments 1, 2 and 3) or not (Experiment 4, abstract condition and Experiment 5, suppression during classification study). This has important implications as it suggests that the attentional modulation observed when retrieving elements serially stored in verbal WM (van Dijck et al., 2013, 2014) is caused by the activation of item-space conjunctions itself and not by a search process over a spatially defined mental representation. On the basis of this, we suggest that semantic information influences order information in the creation of spatial positional markers. The link between items and spatial serial ordering seems also based on the activation of semantic representation associated with these items. Positional models (e.g., Henson, 1998) suggested that item and order information in memory are represented and processed separately. Serial order would be coded by linking each items with an independent representation of the order information. These models are usually embodied by two-layer representations: one layer for the representation of items and the other for the representation of order (i.e., position marker). Because this position marker is considered as a cue to retrieve items in correct order, these models have mainly highlighted the link going from position markers to items. Importantly, our findings suggest an influence of item representation on order representation and so a bidirectional link between both representations (for a similar proposal, see Poirier et al., 2015)

So far, there has been an important debate about domainspecific or domain-general processing for serial order in WM. On the one hand, recent studies support the idea that serial order coding involves domain-specific mechanisms (Saito, Logie, Morita, \& Law, 2008; Soemer \& Saito, 2016). For example, Soemer and Saito (2016) found through the utilization of dualtasks paradigms an interference for serial order performance within modalities but a weak interference between modalities. 
These findings were interpreted as contributions of domainspecific processes for serial order coding. On the other hand, several lines of research suggest a unitary model of serial order coding in WM in which all items (verbal, spatial or visual) share a common representation (for a review see Hurlstone et al., 2014). For instance, supportive evidence for this view comes from a study of Jones et al., (1995) demonstrating similarities between verbal and spatial serial order coding. In line with this last assumption, our data suggest domain-general processes for spatial position markers because the ordinal position effect is led by the activation of semantic networks known to be a unitary and amodal system (e.g., Coccia, Bartolini, Luzzi, Provinciali, \& Ralph, 2004; Jefferies, 2013; Pobric, Jefferies, \& Lambon Ralph, 2010).

In conclusion, this work supports the idea that serial order is coded in a domain general fashion, but suggests that position markers are only spatially coded when the to-be-remembered information is processed at the semantic level.

\section{References}

Abrahamse, E., van Dijck, J.-P., Majerus, S., \& Fias, W. (2014). Finding the answer in space: The mental whiteboard hypothesis on serial order in working memory. Frontiers in Human Neuroscience, 8, 932-944. http:// dx.doi.org/10.3389/fnhum.2014.00932

Acheson, D. J., \& MacDonald, M. C. (2009). Verbal working memory and language production: Common approaches to the serial ordering of verbal information. Psychological Bulletin, 135, 50-68. http://dx.doi .org/10.1037/a0014411

Acheson, D. J., MacDonald, M. C., \& Postle, B. R. (2011). The effect of concurrent semantic categorization on delayed serial recall. Journal of Experimental Psychology: Learning, Memory, and Cognition, 37, 4459. http://dx.doi.org/10.1037/a0021205

Alario, F.-X., \& Ferrand, L. (1999). A set of 400 pictures standardized for French: Norms for name agreement, image agreement, familiarity, visual complexity, image variability, and age of acquisition. Behavior Research Methods, Instruments \& Computers, 31, 531-552. http://dx.doi.org/10 .3758/BF03200732

Awh, E., Jonides, J., \& Reuter-Lorenz, P. A. (1998). Rehearsal in spatial working memory. Journal of Experimental Psychology: Human Perception and Performance, 24, 780-790. http://dx.doi.org/10.1037/00961523.24.3.780

Baddeley, A. (2007). Working memory, thought and action. Oxford, United Kingdom: Oxford University press.

Baddeley, A. (2010). Working memory. Current Biology, 20, R136-R140. http://dx.doi.org/10.1016/j.cub.2009.12.014

Baddeley, A., \& Hitch, G. (1998). Recent developments in working memory. Psychology of Learning and Motivation, 8, 234-238. http://dx.doi .org/10.1016/S0959-4388(98)80145-1

Baddeley, A., \& Logie, R. H. (1999). Working memory: The multiplecomponent model. In A. Miyake \& P. Shah (Eds.), Models of working memory (pp. 28-61). New York, NY: Cambridge University Press.

Barrouillet, P., Plancher, G., Guida, A., \& Camos, V. (2013). Forgetting at short term: When do event-based interference and temporal factors have an effect? Acta Psychologica, 142, 155-167. http://dx.doi.org/10.1016/ j.actpsy.2012.12.003

Bonin, P., Peereman, R., Malardier, N., Méot, A., \& Chalard, M. (2003). A new set of 299 pictures for psycholinguistic studies: French norms for name agreement, image agreement, conceptual familiarity, visual complexity, image variability, age of acquisition, and naming latencies. Behavior Research Methods, Instruments \& Computers, 35, 158-167. http://dx.doi.org/10.3758/BF03195507

Brainard, D. H. (1997). The psychophysics toolbox. Spatial Vision, 10, 433-436. http://dx.doi.org/10.1163/156856897X00357
Burgess, N., \& Hitch, G. J. (2006). A revised model of short-term memory and long-term learning of verbal sequences. Journal of Memory and Language, 55, 627-652. http://dx.doi.org/10.1016/j.jml.2006.08.005

Buzsáki, G., \& Moser, E. I. (2013). Memory, navigation and theta rhythm in the hippocampal-entorhinal system. Nature Neuroscience, 16, 130138. http://dx.doi.org/10.1038/nn.3304

Coccia, M., Bartolini, M., Luzzi, S., Provinciali, L., \& Ralph, M. A. (2004). Semantic memory is an amodal, dynamic system: Evidence from the interaction of naming and object use in semantic dementia. Cognitive Neuropsychology, 21, 513-527. http://dx.doi.org/10.1080/02643290 342000113

Cornoldi, C., \& Vecchi, T. (2004). Visuo-spatial working memory and individual differences. Hove, UK: Psychology Press.

Cowan, N. (1999). An embedded-processes model of working memory. In A. Miyake \& P. Shah (Eds.), Models of working memory: Mechanisms of active maintenance and executive control (pp. 62-101). New York, NY: Cambridge University Press. http://dx.doi.org/10.1017/CBO97811 39174909.006

Darling, S., Della Sala, S., Logie, R. H., \& Cantagallo, A. (2006). Neuropsychological evidence for separating components of visuo-spatial working memory. Journal of Neurology, 253, 176-180. http://dx.doi .org/10.1007/s00415-005-0944-3

Davachi, L., \& DuBrow, S. (2015). How the hippocampus preserves order: The role of prediction and context. Trends in Cognitive Sciences, 19, 92-99. http://dx.doi.org/10.1016/j.tics.2014.12.004

Depoorter, A., \& Vandierendonck, A. (2009). Evidence for modalityindependent order coding in working memory. The Quarterly Journal of Experimental Psychology: Human Experimental Psychology, 62, 531549. http://dx.doi.org/10.1080/17470210801995002

Ebbinghaus, H. (1964). Memory: A contribution to experimental psychology. Mineola, NY: Dover Publications. (Original work published 1885).

Eichenbaum, H. (2014). Time cells in the hippocampus: A new dimension for mapping memories. Nature Reviews Neuroscience, 15, 732-744. http://dx.doi.org/10.1038/nrn3827

Eidswick, J. (2012). A Bayesian alternative to null hypothesis significance testing. Shinken Research Bulletin, 16, 2-15.

Farrell, S., \& Lewandowsky, S. (2002). An endogenous distributed model of ordering in serial recall. Psychonomic Bulletin \& Review, 9, 59-79. http://dx.doi.org/10.3758/BF03196257

Fias, W., Brysbaert, M., \& Geypens, F. (1996). The importance of magnitude information in numerical processing: Evidence from the SNARC effect. Mathematical Cognition, 2, 95-110. http://dx.doi.org/10.1080/ 135467996387552

Ginsburg, V., van Dijck, J.-P., Previtali, P., Fias, W., \& Gevers, W. (2014). The impact of verbal working memory on number-space associations. Journal of Experimental Psychology: Learning, Memory, and Cognition, 40, 976-986. http://dx.doi.org/10.1037/a0036378

Grossberg, S. (1978a). A theory of human memory: Self-organization and performance of sensory-motor codes, maps, and plans. Progress in Theoretical Biology, 5, 233-374.

Grossberg, S. (1978b). Behavioral contrast in short term memory: Serial binary memory models or parallel continuous memory models? Journal of Mathematical Psychology, 17, 199-219. http://dx.doi.org/10.1016/ 0022-2496(78)90016-0

Guida, A., \& Guida-Lavielle, M. (2014). 2011 space odyssey: Spatialization as a mechanism to code order allows a lose encounter between memory expertise and classic immediate memory studies. Frontiers in Psychology: Cognition, 5, 1-5.

Guida, A., Leroux, A., Lavielle-Guida, M., \& Noël, Y. (2016). A SPoARC in the dark: Spatialization in verbal immediate memory. Cognitive Science, 40, 2108-2121.

Henderson, L., \& Matthews, M. L. (1971). Perception and memory loss of item and order information in short-term memory. Perception \& Psychophysics, 9, 231-233. http://dx.doi.org/10.3758/BF03212639 
Henson, R. N. (1998). Short-term memory for serial order: The start-end model. Cognitive Psychology, 36, 73-137. http://dx.doi.org/10.1006/ cogp.1998.0685

Henson, R. N. A., Norris, D., Page, M., \& Baddeley, A. D. (1996). Unchained memory: Error patterns rule out chaining models of immediate serial recall. The Quarterly Journal of Experimental Psychology Section A, 49, 80-115. http://dx.doi.org/10.1080/713755612

Hurlstone, M. J., Hitch, G. J., \& Baddeley, A. D. (2014). Memory for serial order across domains: An overview of the literature and directions for future research. Psychological Bulletin, 140, 339-373. http://dx.doi.org/ $10.1037 / \mathrm{a} 0034221$

Jefferies, E. (2013). The neural basis of semantic cognition: Converging evidence from neuropsychology, neuroimaging and TMS. Cortex: A Journal Devoted to the Study of the Nervous System and Behavior, 49, 611-625. http://dx.doi.org/10.1016/j.cortex.2012.10.008

Jones, D., Farrand, P., Stuart, G., \& Morris, N. (1995). Functional equivalence of verbal and spatial information in serial short-term memory. Journal of Experimental Psychology: Learning, Memory, and Cognition, 21, 1008-1018. http://dx.doi.org/10.1037/0278-7393.21.4.1008

Kalm, K., \& Norris, D. (2014). The representation of order information in auditory-verbal short-term memory. The Journal of Neuroscience, 34, 6879-6886. http://dx.doi.org/10.1523/JNEUROSCI.4104-13.2014

Keuleers, E., \& Brysbaert, M. (2010). Wuggy: A multilingual pseudoword generator. Behavior Research Methods, 42, 627-633. http://dx.doi.org/ 10.3758/BRM.42.3.627

Klauer, K. C., \& Zhao, Z. (2004). Double dissociations in visual and spatial short-term memory. Journal of Experimental Psychology: General, 133, 355-381. http://dx.doi.org/10.1037/0096-3445.133.3.355

Kraus, B. J., Robinson, R. J., II, White, J. A., Eichenbaum, H., \& Hasselmo, M. E. (2013). Hippocampal "time cells": Time versus path integration. Neuron, 78, 1090-1101. http://dx.doi.org/10.1016/j.neuron .2013 .04 .015

Lashley, K. S. (1951). The problem of serial order in behavior. In L. A. Jeffress (Ed.), Cerebral mechanisms in behavior (pp. 112-131). New York, NY: Wiley.

Lewandowsky, S., \& Farrell, S. (2008). Short-term memory: New data and a model. Psychology of Learning and Motivation, 49, 1-48. http://dx .doi.org/10.1016/S0079-7421(08)00001-7

Lorch, R. F., Jr., \& Myers, J. L. (1990). Regression analyses of repeated measures data in cognitive research. Journal of Experimental Psychology: Learning, Memory, and Cognition, 16, 149-157. http://dx.doi.org/ 10.1037/0278-7393.16.1.149

MacDonald, C. J., Carrow, S., Place, R., \& Eichenbaum, H. (2013). Distinct hippocampal time cell sequences represent odor memories in immobilized rats. The Journal of Neuroscience, 33, 14607-14616. http:// dx.doi.org/10.1523/JNEUROSCI.1537-13.2013

Marshuetz, C. (2005). Order information in working memory: An integrative review of evidence from brain and behavior. Psychological Bulletin, 131, 323-339. http://dx.doi.org/10.1037/0033-2909.131.3.323

Marshuetz, C., Smith, E. E., Jonides, J., DeGutis, J., \& Chenevert, T. L. (2000). Order information in working memory: FMRI evidence for parietal and prefrontal mechanisms. Journal of Cognitive Neuroscience, 12(Suppl. 2), 130-144. http://dx.doi.org/10.1162/08989290051137459

McClelland, J. L., \& Rumelhart, D. E. (1981). An interactive activation model of context effects in letter perception: I. An account of basic findings. Psychological Review, 88, 375-407. http://dx.doi.org/10.1037/ 0033-295X.88.5.375

McElree, B., \& Dosher, B. A. (1993). Serial Retrieval processes in the recovery of order information. Journal of Experimental Psychology: General, 122, 291-315. http://dx.doi.org/10.1037/0096-3445.122.3.291
Oberauer, K. (2009). Design for a working memory. Psychology of Learning and Motivation, 51, 45-100. http://dx.doi.org/10.1016/S00797421(09)51002-X

O'keefe, J., \& Nadel, L. (1978). The hippocampus as a cognitive map. New York, NY: Oxford University Press.

Pobric, G., Jefferies, E., \& Ralph, M. A. L. (2010). Amodal semantic representations depend on both anterior temporal lobes: Evidence from repetitive transcranial magnetic stimulation. Neuropsychologia, 48, 1336-1342. http://dx.doi.org/10.1016/j.neuropsychologia.2009.12.036

Poirier, M., Saint-Aubin, J., Mair, A., Tehan, G., \& Tolan, A. (2015). Order recall in verbal short-term memory: The role of semantic networks Memory \& Cognition, 43, 489-499. http://dx.doi.org/10.3758/s13421014-0470-6

Repovš, G., \& Baddeley, A. (2006). The multi-component model of working memory: Explorations in experimental cognitive psychology. Neuroscience, 139, 5-21. http://dx.doi.org/10.1016/j.neuroscience.2005 .12 .061

Rothmayr, C., Baumann, O., Endestad, T., Rutschmann, R. M., Magnussen, S., \& Greenlee, M. W. (2007). Dissociation of neural correlates of verbal and non-verbal visual working memory with different delays Behavioral and Brain Functions, 3, 56. http://dx.doi.org/10.1186/1744 9081-3-56

Saito, S., Logie, R. H., Morita, A., \& Law, A. (2008). Visual and phonological similarity effects in verbal immediate serial recall: A test with kanji materials. Journal of Memory and Language, 59, 1-17. http://dx .doi.org/10.1016/j.jml.2008.01.004

Shapiro, M. L., Kennedy, P. J., \& Ferbinteanu, J. (2006). Representing episodes in the mammalian brain. Current Opinion in Neurobiology, 16, 701-709. http://dx.doi.org/10.1016/j.conb.2006.08.017

Signoret, J. L. (1991). Batterie d'efficience mnésique. Amsterdam, the Netherlands: Elsevier.

Soemer, A., \& Saito, S. (2016). Domain-specific processing in short-term serial order memory. Journal of Memory and Language, 88, 1-17. http://dx.doi.org/10.1016/j.jml.2015.12.003

Sternberg, S. (1967). Retrieval of contextual information from memory. Psychonomic Science, 8, 55-56. http://dx.doi.org/10.3758/BF03330664

Sternberg, S. (1975). Memory scanning: New findings and current controversies. The Quarterly Journal of Experimental Psychology, 27, 1-32. http://dx.doi.org/10.1080/14640747508400459

Stroop, J. R. (1935). Studies of interference in serial verbal reactions Journal of Experimental Psychology, 18, 643-662. http://dx.doi.org/10 $.1037 / \mathrm{h} 0054651$

van Dijck, J.-P., Abrahamse, E. L., Acar, F., Ketels, B., \& Fias, W. (2014) A working memory account of the interaction between numbers and spatial attention. The Quarterly Journal of Experimental Psychology: Human Experimental Psychology, 67, 1500-1513. http://dx.doi.org/10 1080/17470218.2014.903984

van Dijck, J.-P., Abrahamse, E. L., Majerus, S., \& Fias, W. (2013). Spatial attention interacts with serial-order retrieval from verbal working memory. Psychological Science, 24, 1854-1859. http://dx.doi.org/10.1177/ 0956797613479610

van Dijck, J.-P., \& Fias, W. (2011). A working memory account for spatial-numerical associations. Cognition, 119, 114-119. http://dx.doi org/10.1016/j.cognition.2010.12.013

Wagenmakers, E.-J., Wetzels, R., Borsboom, D., \& van der Maas, H. L. J. (2011). Why psychologists must change the way they analyze their data: The case of psi: Comment on Bem (2011). Journal of Personality and Social Psychology, 100, 426-432. http://dx.doi.org/ 10.1037/a0022790 


\section{Appendix}

Illustration of a Concrete Image Used in Experiments 4 and 5 and an Abstract Image Used in Experiment 4<smiles>C1=CCCCCCCCCCC1</smiles>

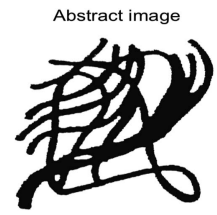

Received October 1, 2014

Revision received December 22, 2016

Accepted December 28, 2016

\section{Correction to Scholten, Read, and Sanborn (2016)}

In the article "Cumulative Weighing of Time in Intertemporal Tradeoffs" by Marc Scholten, Daniel Read, and Adam Sanborn (Journal of Experimental Psychology: General, 2016, Vol. 145, No. 9, pp. 1177-1205. http://dx.doi.org/10.1037/xge0000198), there was an error in Table 1. The preference for faster accumulation read $\{1,000,0,1,000\}>\{0,500,0\}$. It should read $\{0,1,000,0\}>\{500$, $0,500\}$. In addition, in the section Descriptive Accuracy, all the equations with the inequality " $>$ " should read " $\geq$ " instead. The impact of this change is that, when considering the best model for each participant, as measured by Bayes Factors, the absolute goodness of fit, as measured by Bayesian $p$-values, were better than reported in both Table A2 and the text. All of the corrected cells in Table A2 are $0 \%$, meaning that none of the participants across Experiments 2-4 had a significantly ( $p<$ $.05)$ poor fit by the model that described them best. None of the conclusions drawn in the text are altered by this change. 